\title{
Uniqueness of User Equilibrium in Transportation Networks with Heterogeneous Commuters
}

\author{
Hideo Konishi \\ Department of Economics, Boston College, Chestnut Hill, MA 02467, USA. \\ (e-mail) hideo.konishi@bc.edu
}

First Version: March, 2001

Revised: August, 2002 


\title{
Uniqueness of User Equilibrium in Transportation Networks with Heterogeneous Commuters
}

\begin{abstract}
This paper discusses uniqueness of user equilibrium in transportation networks with heterogeneous commuters. Daganzo (1983, Transportation Science) proved the uniqueness of (stochastic) user equilibrium when commuters have heterogeneous tastes over possible paths but identical disutility functions from time costs. We first show, by example, that his result may not apply in general networks if disutility functions are allowed to differ. However, for "simple" transportation networks, we show that user equilibrium is always unique and weakly Pareto efficient ( $c f$. the Braess example) for a general class of utility functions. We investigate if this result applies to more general networks. We also show that user equilibrium is unique in a dynamic bottleneck model with a simple network. We discuss an interesting relationship between the following two problems: the existence of user equilibrium in a finite model and the uniqueness of user equilibrium in a continuum model. In the appendix, we also provide a proof of a slightly generalized version of Daganzo's theorem.
\end{abstract}

Keywords: transportation network, user equilibrium, heterogeneous commuters, uniqueness, efficiency, bottleneck model, game theory 


\section{Introduction}

This paper analyzes the uniqueness of heterogeneous commuters' equilibrium route choices (user equilibrium) in transportation networks. Uniqueness of user equilibrium is important to the transit authority's policy-making on issues such as toll/fare schemes, capacity expansions, or whether to introduce new modes of transportation. If user equilibrium is not unique, the transit authority cannot predict the effects of its policy change. For example, suppose there are two distinct possible user equilibria resulting from an increase in highway tolls: commuters benefit in one user equilibrium, and lose in the other. In such a case, how does the transit authority decide if this policy is beneficial for commuters? If the transit authority arbitrarily assigns a user equilibrium for each policy when it evaluates all possible policies, then the supposedly optimal policy may result in a poor outcome if an unexpected user equilibrium is realized. The same comments apply to the cases of using numerical simulations to evaluate policies when user equilibrium is not unique. Thus it is practically important to identify the situations in which we can guarantee the uniqueness of user equilibrium.

When commuters are homogeneous, uniqueness of user equilibrium is guaranteed under a fairly general condition (see Beckmann et al., 1956, for a potential function approach, and Nagurney, 1993, for a variational inequality approach). As long as congestion is bad for commuters, user equilibrium is unique in static models. However, less is known about the uniqueness of user equilibrium when there are heterogeneous commuters. Although Dafermos (1980) extends the variational inequality analysis to such cases, it is not easy to see what kinds of preferences are allowed in order to secure 
uniqueness. A notable exception is the stochastic user equilibrium literature initiated by Daganzo (1983) (see also Sheffi, 1985). In his pioneering paper, Daganzo (1983) considers an interesting discrete choice model with heterogeneous commuters. He assumes that each commuter's utility from choosing a path is her willingness-to-pay for the path minus her disutility from congestion in the path (i.e., quasi-linear utility functions). Although commuters' disutility functions (interpreted as commuting time) are required to be identical, their willingnesses-to-pay for paths can differ across commuters. He proves that (stochastic) user equilibrium is nonempty and unique in his model.

Although Daganzo's result is obviously helpful, his restriction on preferences may be somewhat strong. First, he requires that commuters have the same relative marginal disutilities among any pair of transportation modes. But consider the following two types of commuters: the first type do not mind waiting (one more minute) in traffic very much as long as they are sitting comfortably in their cars, but they cannot stand being (one more minute) in a congested train. The second type of commuters care only about the amount of time they spend in commuting. Under this kind of situation, the relative marginal disutilities between two transportation modes differ across these two types of commuters. This is not allowed under Daganzo's preference restriction. Second, quite obviously, Daganzo's restriction rules out the possibility that commuters' disutility functions from congestion take different functional forms.

In this paper, we complement Daganzo's (1983) analysis by abandoning his assumption of a common relative marginal disutility. Instead, we impose conditions on the shape of networks in order to guarantee uniqueness of user equilibrium. Through- 
out the paper, we impose three reasonable conditions on commuters' preferences. The first condition is anonymity (AN): Commuters care only about the congestion level of each transportation link: that is, they do not care about characteristics of other commuters. This condition may be questioned by the readers (e.g., car commuters surely care whether the other car commuters are good drivers and bad drivers), but we can weaken this assumption to some extent by using a standard trick in Daganzo (1983) (see concluding section). The second condition is no spillover (NS): Each commuter cares only about the congestion levels of her chosen commuting path (route). The last condition is negative externality from congestion (NEC): A commuter's utility level decreases if her chosen commuting route becomes more congested. As long as these three conditions are satisfied, commuters can be heterogeneous in their tastes in any dimensions and their tastes can differ from each other discretely or continuously. Such a general situation can be modeled easily by using atomless games in game theory. Schmeidler's (1973) classical theorem in atomless games shows that there exists a user equilibrium in pure strategies under anonymity only. Thus the only issue is that of the uniqueness of user equilibrium.

The paper is organized as follows: Section 1 describes the model. Section 2 discusses Daganzo's (1983) result and provides a robust example with multiple user equilibria when his "common relative marginal disutility" assumption is weakened (Example 1). Section 3 shows that if the transportation network is simple, then user equilibrium is essentially unique (Theorem 1) and is efficient in the sense that monetary transfers are not allowed (Theorems 2 and 3 ) under a general class of preferences. A simple network is a network that involves only the origin and the destination 
nodes with multiple disjoint transportation routes (links) connecting these two nodes. Although this restriction is obviously very strong, it may still be a reasonable approximation if the transit authority is interested in commuters' choices over different transportation modes (see, e.g., Arnott and Yan, 2000). Section 4 shows that it is hard to extend these desirable results to cases even with slightly more general networks (Theorem 4, Examples 3 and 4). Section 5 analyzes the dynamic bottleneck model introduced by Vickery (1969) in a simple network. Even though the environment is a bit different (NS does not apply), we can show uniqueness of user equilibrium as an approximation (Theorem 5). Section 6 points out a mysterious and interesting connection between two very different problems: the existence of user equilibrium in a finite model and the uniqueness of user equilibrium in a continuum model. The conditions that guarantee the above two properties are essentially the same. Both Daganzo's restriction on preferences and the restriction of a network being simple are sufficient conditions to obtain positive results in these two problems. Moreover, if these restrictions are dropped, we can find counterexamples to the positive results. Section 7 concludes. In the appendix, we generalize the demand side of Daganzo's theorem, and discuss a potential function approach that can link the two problems in Section 6 .

\section{The Model}

There is a finite set of transportation nodes denoted by $A$. A (multi-mode) transportation network $(A, \mathcal{G})$ is a pair consisting of $A$ and a finite set of links that connect directed pairs of distinct elements of $A$. There can be multiple links 
that directly connect a directed pair of elements in $A$. The set of links that directly connect $a$ with $a^{\prime}$ is denoted by $\mathcal{R}\left(a, a^{\prime}\right) \subset \mathcal{G}$. A path of $(A, \mathcal{G})$ is a sequence of links $\left\{\rho_{1}, \rho_{2}, \ldots, \rho_{K}\right\} \subset \mathcal{G}$ such that there exists a list of $K+1$ distinct nodes $a_{0}, a_{1}, \ldots, a_{K}$, where each $\rho_{k}$ satisfies $\rho_{k} \in \mathcal{R}\left(a_{k-1}, a_{k}\right)$ for $k=1, \ldots, K$. The set of all paths of $(A, \mathcal{G})$ is denoted by $\Omega$. In order to have elastic demand, we can include a "no commuting" option, denoted by $\emptyset$. When this option is included, the choice set for consumers is denoted by $\bar{\Omega} \equiv \Omega \cup\{\emptyset\}$. The reader may wonder if commuters are classified by their origins and destinations (OD) pairs as in the homogeneous commuter literature. However, in the heterogeneous commuter case, such a distinction is not necessary. We can simply assign prohibitively low utilities to infeasible paths (paths with different OD pairs) for commuters. Thus we can use common $\bar{\Omega}$ as commuters' choice set. Since $(A, \mathcal{G})$ is a finite set, $\bar{\Omega}$ is also finite.

There is a continuum of commuters; the set of commuters is given by an interval $I=[0,1]$ and is endowed with the Lebesgue measure $\mu$. An allocation based on commuters' choices is a mapping $f: I \rightarrow \bar{\Omega}$, where $f(i)$ denotes the path that commuter $i$ chooses. The measure of commuters who choose $\omega \in \bar{\Omega}$ under allocation $f$ is denoted by $m_{\omega}(f)=\mu\left(f^{-1}(\omega)\right)$. Thus the distribution of commuters over paths is denoted by a vector $m=m(f)=\left(m_{\omega}(f)\right)_{\omega \in \bar{\Omega}} \in M=\mu(I) \times \triangle^{\bar{\Omega}}\left(\triangle^{\bar{\Omega}}\right.$ represents a $|\bar{\Omega}|$ dimensional simplex). Each commuter makes her choice over the available paths depending on her utility function $u$. The space of utility functions (commuters' characteristics) is denoted by $\mathcal{U}$. The economy is described by a measurable mapping $e: I \rightarrow \mathcal{U}$, where $e(i)=u \in \mathcal{U}$ denotes commuter $i$ 's utility function. Her utility is dependent on her own path and on other commuters' choices of paths (defined formally below under 
the case of anonymity). For each $u \in \mathcal{U}$, we can find the measure of commuters whose utility function is $u$ by $\mu\left(e^{-1}(u)\right)$. Every commuter's utility function is assumed to satisfy the following condition.

Anonymity (AN): Suppose that allocations $f$ and $g$ attain the same distribution of commuters over paths: $m(f)=m(g)$. Then, for any $u \in \mathcal{U}, u$ attains the same utility levels for $f$ and $g$ as long as the path chosen by the commuter is the same. In other words, utility function $u \in \mathcal{U}$ can be written as a function $u: \bar{\Omega} \times M \rightarrow \Re$ such that $u=u(\omega, m)$ denotes a commuter's utility by choosing path $\omega$ when the distribution of commuters is described by $m$. We set $u(\emptyset, m)=u(\emptyset)$.

This assumption says that each commuter cares only about how many others choose each path: she does not care who they are. For notational simplicity, we denote $e(i)$ by $u_{i}$ from now on.

To introduce more preference restrictions, we need more notation. The measure of commuters who use link $\rho$ is described by $\left.n_{\rho}(m) \equiv \sum_{\omega \ni \rho} m_{\omega}\right)$, since mode $\rho$ is utilized by commuters who choose paths $\omega$ s that contain $\rho$ as a part of them. We impose more assumptions on utility functions:

No Spillovers (NS): For any $u \in \mathcal{U}$, any $\omega \in \Omega$, any $\rho \in \omega$, and any $m, m^{\prime} \in M$ with $n_{\rho}(m)=n_{\rho}\left(m^{\prime}\right), u(\omega, m)=u\left(\omega, m^{\prime}\right)$ follows.

This assumption means that each commuter cares only about the congestion levels of the links in her chosen path. It is commonly used in the literature, although it assumes there are no delays in intersections (nodes). The final assumption builds on 
NS:

Negative Externality from Congestion (NEC): For any $u \in \mathcal{U}$, any $\omega \in \Omega$, and any $m, m^{\prime} \in M$ such that $n_{\rho}(m) \leq n_{\rho}\left(m^{\prime}\right)$ holds for any $\rho \in \omega$ with at least one of the inequalities being strict, $u(\omega, m)>u\left(\omega, m^{\prime}\right)$ follows.

The meaning of this assumption is that a commuter's utility level decreases as the number of commuters who choose the same links as she chooses increases. This assumption does not seem questionable in the transportation problem. It says only that commuters do not want congestion. For obvious reasons, we do not require strict inequality for no commuting $(\emptyset)$.

We now define a user equilibrium allocation. A user equilibrium allocation is an allocation $f$ such that commuter $i$ chooses her optimal transportation path $a$ almost everywhere (a.e.) for $i$ in $I$ : i.e., $u_{i}(f(i), m(f)) \geq u_{i}(\omega, m(f))$ for any $\omega \in \bar{\Omega}$, where $u_{i}=e(i)$. Commuter $i$ chooses transportation path $f(i)=\omega^{\prime}$ only if $u_{i}\left(\omega^{\prime}, m\right) \geq u_{i}(\omega, m)$ for any $\omega \in \bar{\Omega}$.

In order to guarantee the existence of user equilibrium, we assume that $u$ satisfies a regularity assumption: (i) $u(\omega, \cdot): M \rightarrow \Re$ is continuous for any $u \in \mathcal{U}$, and there is a constant $K$ such that $|u(\omega, m)|<K$ for any $u \in \mathcal{U}$, any $\omega \in \bar{\Omega}$, and any $m \in M$, and (ii) the set $\left\{i \in I: u_{i}(\omega, m)>u_{i}\left(\omega^{\prime}, m\right)\right\}$ is measurable for any $u \in \mathcal{U}$, any $\omega, \omega^{\prime} \in \bar{\Omega}$, and any $m \in M$. This technical assumption is due to Schmeidler (1973) and will be adopted throughout the paper. The following is the fundamental result in anonymous games obtained by Schmeidler (1973). 
Theorem. (Schmeidler, 1973) Under AN, there exists a user equilibrium allocation.

We can define the essential uniqueness of user equilibrium with AN and NS. We say that user equilibrium allocation is essentially unique if and only if at any user equilibria $f$ and $g, n_{\rho}(m(f))=n_{\rho}(m(g))$ follows for any $\rho \in \mathcal{G}$. Note that with NS, if the congestion level at each link is the same under $f$ and $g$, then we necessarily have $u_{i}(f(i), m(f))=u_{i}(g(i), m(g))$ for $i$ a.e. in $I$. In the next section, we show that user equilibrium is not essentially unique in general networks.

\section{Nonuniqueness of User Equilibrium}

In this section, we demonstrate the nonuniqueness of user equilibrium if Daganzo's (1983) preference restriction is weakened. We first review Daganzo's result, and then we proceed to the example.

\subsection{Daganzo's result}

Daganzo assumes the following quasi-linear utility function (cost function is simplified here). Commuter $i$ 's utility function when she uses $\omega$ is written as:

$$
u_{i}(\omega, m)=\sum_{\rho \in \omega} v_{i}\left(\rho, n_{\rho}(m)\right)=\sum_{\rho \in \omega}\left(\theta_{\rho i}-c_{\rho}\left(n_{\rho}(m)\right)\right)
$$

where $\theta_{\rho i}$ and $c_{\rho}\left(n_{\rho}\right)$ denote commuter $i$ 's personal taste on link $\rho$ and common congestion cost (commuting time) in link $\rho$, respectively. Thus commuters are allowed to differ only with respect to their $\theta_{i} \mathrm{~s}\left(\theta_{i} \equiv\left(\theta_{\rho i}\right)_{\rho \in \mathcal{G}}\right)$. When $\omega=\emptyset, u_{i}(\emptyset)=v_{i}(\emptyset)$ 
and $c_{\emptyset}(n)=0$. The economy is specified by a distribution of $\theta$. Under the law of large numbers (see Judd, 1985, for the case of a continuum of IID random variables), consumers' taste distribution and the distribution of consumers' random utilities become equivalent, and the Daganzo model can be regarded as a type of discrete choice model (for a nice survey of discrete choice models, see Anderson, de Palma, and Thisse, 1992). As is easily seen, this model is a direct generalization of the standard homogeneous commuter model in which commuters care only about costs, $-\sum_{\rho \in \omega} c_{\rho}\left(n_{\rho}(m)\right)$. With this preference restriction, Daganzo (1983) proves the following theorem. This version of Daganzo's theorem is slightly more general than the original one in terms of distribution of commuters' tastes. Although the line of the proof is essentially the same (both use "strict monotonicity"), we need no restriction over the distribution of $\theta$ (we drop his Assumption 2). We provide a proof in the appendix for completeness.

Theorem. (Daganzo, 1983) Suppose that commuters' utility functions have a quasilinear form with common congestion costs: i.e., $u_{i}(\omega, m)=\sum_{\rho \in \omega}\left(\theta_{\rho i}-c_{\rho}\left(n_{\rho}(m)\right)\right)$. If $c_{\rho} \mathrm{s}$ are continuous and strictly increasing, then user equilibrium is essentially unique.

Although Daganzo's result is useful, it does not apply to the case where commuters have heterogenous relative marginal disutilities. Obviously, it is a very strong condition to impose that the functional forms (the shapes) of $c_{\rho}$ s are exactly the same among commuters. However, even a slight modification of the utility functions can cause a problem. Consider the following functional form:

$$
u_{i}(\omega, m)=\sum_{\rho \in \omega} v_{i}\left(\rho, n_{\rho}(m)\right)=\sum_{\rho \in \omega}\left(\theta_{\rho i}-\phi_{\rho i} \times c_{\rho}\left(n_{\rho}(m)\right)\right)
$$


where a positive vector $\phi_{i} \equiv\left(\phi_{\rho i}\right)_{\rho \in \mathcal{G}}$ represents commuter $i$ 's marginal disutility vector. The second term in the parenthesis $\phi_{\rho i} \times c_{\rho}\left(n_{\rho}(m)\right)$ denotes the amount of disutility commuter $i$ suffers in going through link $\rho$. In this case, the functional forms of $c_{\rho} \mathrm{s}$ are still identical: only coefficients of $c_{\rho} \mathrm{s}$ are heterogeneous among commuters. Note that as long as $\phi_{i}$ and $\phi_{j}$ are proportional to each other for any $i$ and $j$, we can apply Daganzo's argument. We need only normalize $\phi_{i}$ s by multiplying a scalar to $\theta_{i}$ and $\phi_{i}$. Such a situation $\left(\phi_{i}=t \times \phi_{j}\right.$ for each pair of $i$ and $\left.j\right)$ corresponds to the case where commuters are different only in their time costs (opportunity costs: wage rates). By redefining $\tilde{\theta}_{i}=\theta_{i} / t$ and $\tilde{c}_{\rho}=\phi_{j} c_{\rho}$, we can recover Daganzo's functional form. Arnott, de Palma, and Lindsey (1992), Verhoef and Small (1999), and Small and Yan (1999) assume that commuters are heterogeneous in their time costs and analyze the optimal pricing rules (at various levels) over two different transportation modes. These papers adopt proportionality of $\phi_{i} \mathrm{~s}$.

However, if $\phi_{i}$ s are not proportional to each other, then we can no longer apply Daganzo's argument. Recall the example discussed above in the introduction. Suppose that there are two types of commuters: type 1 commuters do not mind spending one more minute in traffic very much as long as they are sitting comfortably in their car, but they cannot stand being in a congested train for one more minute. Type 2 commuters primarily care about the amount of time spent in commuting. In such a situation, the ratio of $\phi_{c a r 1}$ and $\phi_{t r a i n 1}$ and the one of $\phi_{c a r 2}$ and $\phi_{\operatorname{train} 2}$ are very different from each other, thus violating Daganzo's preference restriction. Example 1 in the next subsection shows that user equilibrium may be multiple if this preference restriction is violated. 


\subsection{An Example}

In this subsection, we show that there can be multiple user equilibria if Daganzo's preference restriction is slightly weakened. This counterexample seems robust. The transportation network is not complicated, congestion cost is linear, demand is inelastic (there is no noncommuting option), and all types of commuters have the same origins and destinations.

EXAmple 1. There is a transportation network connecting four nodes $\{a, b, c, d\}$ with six links: $\rho_{1}(a \rightarrow b), \rho_{2}(b \rightarrow d), \rho_{3}(b \rightarrow d), \rho_{4}(a \rightarrow c), \rho_{5}(c \rightarrow d), \rho_{6}(c \rightarrow d)$ (see Figure 1-1). There are three types of atomless commuters (types 1, 2, and 3) commuting from node $a$ to node $d$. The measures of types 1,2 , and 3 are $1.2,1$, and 1 , respectively. There are four paths $\Omega=\left\{\omega_{1}, \omega_{2}, \omega_{3}, \omega_{4}\right\}$, where $\omega_{1}=\left(\rho_{1}, \rho_{2}\right)$, $\omega_{2}=\left(\rho_{1}, \rho_{3}\right), \omega_{3}=\left(\rho_{4}, \rho_{5}\right)$, and $\omega_{4}=\left(\rho_{4}, \rho_{6}\right)$. There is no noncommuting option. Each commuter's utility function is additively separable over links: $\sum_{\rho \in \omega} v_{i}\left(\rho, n_{\rho}\right)=$ $\sum_{\rho \in \omega}\left(\theta_{\rho i}-\phi_{\rho i} c_{\rho}\left(n_{\rho}\right)\right)$ with $c_{\rho}\left(n_{\rho}\right)=n_{\rho}$ for each $\rho$. Three types of commuters have the following subutility functional forms, respectively (we omit subscript $\rho$ from $n_{\rho}$ for simplicity):

Type 1:

$$
\begin{aligned}
& v_{1}\left(\rho_{1}, n\right)=v_{1}\left(\rho_{2}, n\right)=v_{1}\left(\rho_{4}, n\right)=v_{1}\left(\rho_{6}, n\right)=-n, \\
& v_{1}\left(\rho_{3}, n\right)=v_{1}\left(\rho_{5}, n\right)=-100-n
\end{aligned}
$$


Type 2:

$$
\begin{aligned}
& v_{2}\left(\rho_{1}, n\right)=v_{2}\left(\rho_{4}, n\right)=-n, \\
& v_{2}\left(\rho_{2}, n\right)=19-20 n, \\
& v_{2}\left(\rho_{5}, n\right)=-2-n, \\
& v_{2}\left(\rho_{3}, n\right)=v_{2}\left(\rho_{6}, n\right)=-100-n ;
\end{aligned}
$$

Type 3:

$$
\begin{aligned}
& v_{3}\left(\rho_{1}, n\right)=v_{3}\left(\rho_{4}, n\right)=-n, \\
& v_{3}\left(\rho_{6}, n\right)=19-20 n \\
& v_{3}\left(\rho_{3}, n\right)=-2-n, \\
& v_{3}\left(\rho_{2}, n\right)=v_{2}\left(\rho_{5}, n\right)=-100-n .
\end{aligned}
$$

Claim 1. Example 1 possesses two distinct equilibria in which everybody in the same type chooses the same path. In user equilibrium (A), type 1 commuters choose $\omega_{1}$, and types 2 and 3 commuters choose $\omega_{3}$ and $\omega_{4}$, respectively. In user equilibrium (B), types 1,2 , and 3 choose $\omega_{4}, \omega_{1}$, and $\omega_{2}$, respectively.

Proof. First observe that types 1, 2, and 3 choose from sets of paths $\left\{\omega_{1}, \omega_{4}\right\}$, $\left\{\omega_{1}, \omega_{3}\right\}$, and $\left\{\omega_{2}, \omega_{4}\right\}$, respectively. This is because type 1 (2 and 3$)$ will not choose links $\rho_{3}$ and $\rho_{5}\left(\rho_{3}\right.$ and $\rho_{6}$; and $\rho_{2}$ and $\left.\rho_{5}\right)$ given that the total measure of population is 3.2. We prove that the allocation described as user equilibrium (A) is actually a 
user equilibrium. A similar argument applies for user equilibrium (B) almost symmetrically. In allocation (A), the congestion level at each link is described as follows: $n_{\rho_{1}}=n_{\rho_{2}}=1.2, n_{\rho_{3}}=0, n_{\rho_{4}}=2$, and $n_{\rho_{5}}=n_{\rho_{6}}=1$. We start with type 1 commuters. On path $\omega_{1}$, a type 1 commuter obtains -2.4 , but if she switches to $\omega_{4}$, then she obtains only -3 . Thus no type 1 commuter has an incentive to switch her path. Second, consider type 2 commuters. In allocation (A), a type 2 commuter obtains -5 . If she switches to $\omega_{1}$, then she obtains -6.2 . Similarly, no type 3 commuter has an incentive to switch her path either. In allocation (A), she obtains -3 , yet she gets only -3.2 by choosing $\omega_{2}$. Thus allocation (A) is a user equilibrium. It is easy to show that allocation (B) is also a user equilibrium owing to symmetry. Thus the user equilibrium (A) payoff vector is $(-2.4,-5,-3)$, and the user equilibrium (B) payoff vector is $(-2.4,-3,-5)$

A couple of remarks are in order. First, note that $\phi_{i}$ s are common in most links: $\phi_{1}=(1,1,1,1,1,1), \phi_{2}=(1,20,1,1,1,1)$, and $\phi_{3}=(1,1,1,1,1,20)$. Only two links have heterogeneous $\phi_{i}$ s. This implies that Daganzo's result is sensitive to the preference restriction. Second, commuters' utilities differ between these two equilibria. Types 2 and 3 have different utilities in (A) and (B) ( -5 and -3 , and -3 and -5 , respectively). Although type 1 commuters have the same utilities in this example, we can make them strictly prefer user equilibrium (B) by modifying their payoff function slightly: i.e., $v_{1}\left(\rho_{1}, n\right)=-0.1-n$. We assume symmetry only to give a clearer example. Third, these two user equilibria are both locally stable in a natural sense (although we do not provide formal stability analysis). Even if small numbers (mea- 
sures) of any types of consumers deviate from a user equilibrium, they will revise their strategies and recover the original user equilibrium. Thus it is very difficult to select a more "natural" user equilibrium among these two. For an analysis of the evolutionary stability of user equilibrium, see Sandholm (2001a). Sandholm analyzes homogeneous commuter case using a potential function approach (see the appendix: see also Tabuchi and Zeng, 2001). Finally, note that we can easily make Example 1 more realistic without altering the result, although its simplicity and clarity might be compromised (available from the author upon request).

\section{Simple Networks}

In this section, we impose a strong condition on the transportation network $\mathcal{G}$. A transportation network $(A, \mathcal{G})$ is simple if and only if $A=\left\{a, a^{\prime}\right\}$ and $\Omega=\mathcal{R}\left(a, a^{\prime}\right)=$ $\mathcal{G}$, and $\bar{\Omega}=\Omega \cup\{\emptyset\}$. That is, there are only two transportation nodes, the origin and the destination, in a simple network (see Figure 2). The implication of assuming a simple network is that each commuter's utility depends only on her choice of $\omega$ and the number of commuters who choose the same $\omega$. Obviously, Example 1 does not satisfy this condition. Given this restriction, we can abuse our notation a little bit: we can denote the utility function by $u_{i}\left(\omega, m_{\omega}\right)$ instead of $u_{i}(\omega, m)$. This condition guarantees two desirable properties of user equilibrium: uniqueness and efficiency.

\subsection{Uniqueness}

We start with uniqueness: 
Theorem 1. Suppose that $(A, \mathcal{G})$ is simple and that there are commuting options. Under AN, NS, and NEC, user equilibrium allocation is essentially unique.

Proof. Suppose that $f$ and $g$ are both user equilibrium allocations with $m(f) \neq m(g)$. Then, without loss of generality, there is $\omega_{0} \in \Omega\left(\omega_{0} \neq \emptyset\right)$ such that $m_{\omega_{0}}(f)<m_{\omega_{0}}(g)$. Thus there is a subset $C_{0} \subset I$ with $\mu\left(C_{0}\right)>0$ and $\omega_{0}=g(i) \neq f(i)$ for $i$ a.e. in $C_{0}$. Given NEC, $u_{i}\left(\omega_{0}, m_{\omega_{0}}(g)\right)<u_{i}\left(\omega_{0}, m_{\omega_{0}}(f)\right)$. Since $f$ is a user equilibrium allocation, $u_{i}\left(\omega_{0}, m_{\omega_{0}}(f)\right) \leq u_{i}\left(f(i), m_{f(i)}(f)\right)$ for $i$ a.e. in $C_{0}$. On the other hand, since $g$ is a user equilibrium allocation, $u_{i}\left(f(i), m_{f(i)}(g)\right) \leq u_{i}\left(\omega_{0}, m_{\omega_{0}}(g)\right)$ for $i$ a.e. in $C_{0}$. Taking these three inequalities together, we obtain $u_{i}\left(f(i), m_{f(i)}(g)\right)<u_{i}\left(f(i), m_{f(i)}(f)\right)$ for $i$ a.e. in $C_{0}$. Thus we can conclude that there is a path $\omega_{1} \in \Omega \backslash\left\{\omega_{0}\right\}$ with $m_{\omega_{1}}(f)<m_{\omega_{1}}(g)$ (set $\omega_{1}$ to be such $f(i)$ ). By repeating the same argument, we obtain a sequence $\left\{\omega_{0}, \omega_{1}, \ldots\right\}$ such that for each $k, m_{\omega_{k}}(f)<m_{\omega_{k}}(g)$. By construction, for any positive integer $k$, there are $\omega_{k+1} \in \Omega \backslash\left\{\omega_{0}, \ldots, \omega_{k}\right\}$. However, since $\Omega$ is a finite set, this is not possible, and we have a contradiction. Hence $m(f)=m(g)$ for any user equilibrium allocations $f$ and $g$

The idea behind the proof is very simple. If there are two distinct user equilibrium allocations $f$ and $g$, then there is at least one path that is strictly less congested under $f$ (without loss of generality). This implies that under $g$ more commuters use this path. Since $f$ is a user equilibrium, those commuters who decide not to use this path (emigrés) voluntarily choose other paths if user equilibrium switches from $g$ to $f$. However, this means that these other paths are also less congested under $f$ than under $g$, since otherwise emigrés would not have moved out of the original path (it 
was the best choice for them under $g$, and it is less congested under $f$ ). Also note that commuters would not switch to noncommuting option, since it could have been chosen under $g$. By applying the same argument repeatedly, we reach a contradiction, since there is only a finite number of paths and the population is fixed. Actually, Theorem 1 is a variation of Milchtaich (2000). He shows that in a class of atomless congestion games, the user equilibrium distribution of strategies is generically unique even under equilibria with mixed strategies. Our proof is much more elementary since we concentrate on the pure strategy case. We also include a noncommuting option.

Congestible transportation modes are the crucial property for the result of Theorem 1. The following example illustrates the importance of NEC.

Example 2. (network externalities) Consumers are homogeneous $(\{u\}=\mathcal{U})$, and $\Omega=\{\alpha, \iota\}$ (they stand for Apple and IBM). The utility function is described by $u\left(\alpha, m_{\alpha}\right)=0.5+m_{\alpha}$ and $u\left(\iota, m_{\iota}\right)=m_{\iota}$. Note that NEC is violated here. In this example, there are three user equilibrium distributions: $\left(m_{\alpha}, m_{\iota}\right)=(1,0),(0.25,0.75)$, and $(0,1)$. These equilibria are strictly Pareto ranked, and $(1,0)$ is the Pareto efficient user equilibrium. The reason there are multiple Nash equilibria is that externalities are positive instead of negative (there are network externalities instead of NEC).

\subsection{Efficiency}

In this subsection, we discuss the efficiency of user equilibrium. As is well known, a user equilibrium allocation is not efficient when appropriate transfers among consumers and the government are available (as with, say, Pigouvian tax). The proposed notion of efficiency is a constrained efficiency in the sense that the transfer of money 
is prohibited. Interestingly, the simple network assumption also guarantees efficiency of user equilibrium (in the heterogeneous commuter case). Even in the homogeneous commuter case, a nonsimple network may have a unique inefficient user equilibrium in the sense that there is an allocation that is strictly better than the user equilibrium allocation. To see this, we can simply recall a famous and beautiful example by Braess (1968; see, e.g., Nagurney, 1993). It shows that building a bridge may reduce commuters' equilibrium utilities in the homogeneous commuter case. This implies that user equilibrium in the network with the bridge is inefficient: an allocation without using the bridge Pareto dominates the user equilibrium.

The Braess example implies that the user equilibrium allocation may not be efficient when the transportation network $(A, \mathcal{G})$ is not simple. The unique user equilibrium distribution $m$ attains a lower utility level than a user distribution $m^{\prime}$ that is obviously feasible in the original network. This implies that user equilibrium may not be even weakly Pareto efficient in general. Formally, a weakly Pareto efficient allocation is an allocation $f$ such that there is no allocation $g$ such that for almost any $i \in I$, $u_{i}(g(i), m(g))>u_{i}(f(i), m(f))$. A Pareto efficient allocation is an allocation $f$ such that there is no allocation $g$ with $u_{i}(g(i), m(g)) \geq u_{i}(f(i), m(f))$ a.e. in $i \in I$, and there is a subset $C \subseteq I$ with $\mu(C)>0$ such that $u_{i}(g(i), m(g))>u_{i}(f(i), m(f))$ a.e. in $i \in C$. Note that our definition of (weak) Pareto efficiency is defined only over allocations without transfers among commuters. Apparently, a Pareto efficient allocation is also a weakly Pareto efficient allocation. In simple networks, we can show that user equilibrium allocations are weakly Pareto efficient even when commuters are heterogeneous. 
Theorem 2. Suppose that $(A, \mathcal{G})$ is simple and that there are noncommuting options. Under AN, NS, and NEC, a user equilibrium allocation is weakly Pareto efficient.

Proof. Let $f$ be a user equilibrium. Then, for any $\omega \in \bar{\Omega}, u_{i}\left(f(i), m_{f(i)}(f)\right) \geq$ $u_{i}\left(\omega, m_{\omega}(f)\right)$ follows for $i$ a.e. in $I$. Now, suppose that there is an allocation $g$ that Pareto dominates $f$. Then $u_{i}\left(f(i), m_{f(i)}(f)\right)<u_{i}\left(g(i), m_{g(i)}(g)\right)$ follows for $i$ a.e. in I. This implies that $u_{i}\left(g(i), m_{g(i)}(g)\right)>u_{i}\left(g(i), m_{g(i)}(f)\right)$ for $i$ a.e. in $I$. Since $(A, \mathcal{G})$ is simple, NEC implies $m_{g(i)}(g)<m_{g(i)}(f)$ for $i$ a.e. in $I$. Since the total population is fixed, $\sum_{\omega \in \bar{\Omega}} m_{\omega}(f)=\sum_{\omega \in \bar{\Omega}} m_{\omega}(g)$. This is a contradiction.

This theorem can be considered a corollary of Konishi, Le Breton, and Weber's (1997a) Proposition 4.4. Proposition 4.4 shows that if $f$ is a user equilibrium, then it is also a strong Nash user equilibrium under AN, NS, and NEC: i.e., there is no measurable subset $C$ with $\mu(C)>0$ and no allocation $g: I \rightarrow \bar{\Omega}$ with $g(i)=f(i)$ for $i$ a.e. in $I \backslash C$ such that $u_{i}\left(g(i), m_{g(i)}(g)\right)>u_{i}\left(f(i), m_{f(i)}(f)\right)$ for $i$ a.e. in $C$. Thus, by setting $C=I$, we can show weak Pareto efficiency. Here, we provided a direct and simple proof instead. A natural next question is if we can strengthen the notion of efficiency. The answer is unfortunately "no" even when commuters are homogeneous.

Example 3. Commuters are homogeneous $(\{u\}=\mathcal{U})$, and $\bar{\Omega}=\{\omega, \emptyset\}$. The set of consumers $I=[0,1]$ is endowed with Lebesgue measure. The utility function is described by $u\left(\emptyset, m_{\emptyset}\right)=0.5$ and $u\left(\omega, m_{\omega}\right)=1-m_{\omega}$. Then, the unique user 
equilibrium distribution is $\left(m_{0}, m_{\omega}\right)=(0.5,0.5)$. A user equilibrium allocation can be written as $f: I \rightarrow \bar{\Omega}$ such that $f(i)=\emptyset$ if $i \in[0,0.5]$ and $f(i)=\omega$ if $i \in(0.5,1]$. For $i$ a.e. in $I, u_{i}=0.5$ in the user equilibrium. However, this is not a Pareto efficient choice. Consider $g_{\epsilon}: I \rightarrow \bar{\Omega}$ such that $g_{\epsilon}(i)=\emptyset$ if $i \in[0,0.5+\epsilon)$ and $g_{\epsilon}(i)=\omega$ if $i \in[0.5+\epsilon, 1]$. In this case, $u_{i}=0.5$ for $i \in[0,0.5+\epsilon)$, but $u_{i}=0.5+\epsilon$ for $i \in[0.5+\epsilon, 1]$ for any $\epsilon \in(0,0.5)$.

This example is quite robust, so given a noncommuting option, we cannot expect Pareto efficiency of user equilibrium allocation. However, it is weakly Pareto efficient, and it is on the boundary of the set of Pareto efficient allocations $\left(\lim _{\epsilon \rightarrow 0} g_{\epsilon}=f\right)$. One of the reasons Pareto efficiency is not attained is that user equilibrium requires equal treatment of equals. Thus one can expect that if commuters who are indifferent between not commuting and commuting form a zero measure, the efficiency result can be recovered. The following Theorem summarizes the results.

Theorem 3. Suppose that $(A, \mathcal{G})$ is simple. Under AN, NS, and NEC, if either (i) there is no noncommuting option $(\Omega$ instead of $\bar{\Omega})$ or (ii) a user equilibrium allocation satisfies $\mu\left(\left\{i \in I: u_{i}\left(f(i), m_{f(i)}(f)\right)=u_{i}(\emptyset)\right\}\right)=0$, then the user equilibrium is Pareto efficient.

Before we provide the proof, we prove the following lemma:

Lemma 1. Suppose that $(A, \mathcal{G})$ is simple. Suppose that AN, NS, and NEC are satisfied. Moreover, we assume either condition (i) or (ii) in Theorem 3 holds. If $g$ is Pareto superior choice to a user equilibrium allocation $f$, then $m_{\omega}(f)=m_{\omega}(g)$ holds 
for each $\omega \in \bar{\Omega}$.

Proof. First, we show that $m_{\omega}(g)>m_{\omega}(f)=0$ cannot happen. Suppose to the contrary that it is the case. Then, commuter $i$ weakly prefers $g$ to $f$ a.e. in $g^{-1}(\omega)$. This is equivalent to: $u_{i}\left(\omega, m_{\omega}(g)\right) \geq u_{i}\left(f(i), m_{f(i)}(f)\right)$ a.e. in $g^{-1}(\omega)$. If $\omega \neq \emptyset$ (condition (i)), then, by NEC, $u_{i}(\omega, 0)>u_{i}\left(\omega, m_{\omega}(g)\right)$ holds. This, together with our supposition, implies that $u_{i}\left(\omega, m_{\omega}(f)\right)=u_{i}(\omega, 0)>u_{i}\left(f(i), m_{f(i)}(f)\right)$ a.e. in $g^{-1}(\omega)$. This is a contradiction to $f$ being a user equilibrium. If $\omega=\emptyset$ and $\mu(\{i \in$ $\left.\left.I: u_{i}\left(f(i), m_{f(i)}(f)\right)=u_{i}(\emptyset)\right\}\right)=0($ condition (ii)), then there are a subset $C \subset I$ with $\mu(C)>0$ and a path $\omega^{\prime} \neq \emptyset$ such that $f(i)=\omega^{\prime}$ and $g(i)=\emptyset$ a.e. in $C$. Since $f$ is a user equilibrium allocation and since $\mu\left(\left\{i \in I: u_{i}\left(f(i), m_{f(i)}(f)\right)=u_{i}(\emptyset)\right\}\right)=0$, $u_{i}\left(\omega^{\prime}, m_{\omega^{\prime}}(f)\right)>u_{i}(\emptyset)$. This is a contradiction to $g$ being Pareto-superior to $f$.

Second, we show that $m_{\omega}(g)=m_{\omega}(f)$ has to hold for any $\omega$ with $m_{\omega}(f)>0$. Suppose not. Then, there is $\omega$ such that $m_{\omega}(g)>m_{\omega}(f)$. This implies that there is a subset $S \subset C$ with $\mu(S)>0$ such that almost everywhere in $S$, commuter $i$ chooses $\omega$ under $g$, but does not choose $\omega$ under $f$. Suppose first that $\omega \neq \emptyset$. In this case, by the same logic as in the previous case, $u_{i}\left(\omega, m_{\omega}(f)\right)>u_{i}\left(\omega, m_{\omega}(g)\right) \geq u_{i}\left(f(i), m_{f(i)}(f)\right)$ holds. This is a contradiction. Suppose second that $\omega=\emptyset$. Then, there is a subset $T \subset I$ with $\mu(T)>0$ and $\omega^{\prime} \neq \emptyset$ such that almost everywhere in $T$, commuter $i$ chooses $\omega^{\prime}$ under $f$ and does not choose $\omega^{\prime}$ under $g$ (since there is $\omega^{\prime} \neq \emptyset$ with $m_{\omega^{\prime}}(f)>m_{\omega^{\prime}}(g)$ owing to constant total measure). By applying the same argument as before, we again get a contradiction, and the proof of Lemma 1 is complete.

Proof of Theorem 3. We show that there is no allocation $g$ that Pareto dominates 
a user equilibrium choice $f$. Suppose that there is such an allocation $g$. Then, there is a $C \subset I$ with $\mu(C)>0$ and $g: I \rightarrow \bar{\Omega}$ such that $u_{i}\left(g(i), m_{g(i)}(g)\right)>u_{i}\left(f(i), m_{f(i)}(f)\right)$ a.e. in $C$, and $u_{i}\left(g(i), m_{g(i)}(g)\right)=u_{i}\left(f(i), m_{f(i)}(f)\right)$ a.e. in $I \backslash C$. Since preference inequalities are strict a.e. in $C, g(i) \neq f(i)$ a.e. in $C$. However, from Lemma $1, m_{\omega}(g)=m_{\omega}(f)$ for any $\omega \in \bar{\Omega}$. This implies that under $f$, these commuters in $C$ had incentives to choose $g(i)$, since $u_{i}\left(g(i), m_{g(i)}(g)\right)=u_{i}\left(g(i), m_{g(i)}(f)\right)>$ $u_{i}\left(f(i), m_{f(i)}(f)\right)$ a.e. in $C$. This is a contradiction. Therefore, any user equilibrium allocation $f$ must be Pareto efficient.

\section{Node-Tree Networks}

In this section, we explore whether our results in the previous section extend to more general cases. We first define a node-tree network that is a natural extension of a simple network. Given node-tree networks, we consider two cases: inelastic demand (without a noncommuting option) and elastic demand case (with a noncommuting option). This distinction turns out to be critical in the general case, although it played no role in establishing uniqueness in the simple network case. We start with imposing restrictions on the transportation network and utility functions in order to guarantee uniqueness of user equilibrium.

First, we need a few definitions. In order to guarantee a positive result, we will not allow noncommuting options in the commuters' choice set. In this particular case, it is convenient to specify the origin and the destination of each commuter. The set of commuters can be partitioned according to their origins and destinations. Let $I\left(a, a^{\prime}\right) \subset I$ for $a \neq a^{\prime}$ be the set of commuters who commute from node $a$ to node $a^{\prime}$. 
Obviously, $I=\cup_{a, a^{\prime} \in A, a \neq a^{\prime}} I\left(a, a^{\prime}\right)$ and $I\left(a, a^{\prime}\right) \cap I\left(a^{\prime \prime}, a^{\prime \prime \prime}\right)=\emptyset$ if $a \neq a^{\prime \prime}$ or $a^{\prime} \neq a^{\prime \prime \prime}$. The relevant paths in $\Omega$ for $i \in I\left(a, a^{\prime}\right)$ are just those that start from $a$ and end at $a^{\prime}$. We denote the collection of all such paths by $\Omega\left(a, a^{\prime}\right)$. Thus $\Omega=\cup_{a, a^{\prime} \in A, a \neq a^{\prime}} \Omega\left(a, a^{\prime}\right)$ and $\Omega\left(a, a^{\prime}\right) \cap \Omega\left(a^{\prime \prime}, a^{\prime \prime \prime}\right)=\emptyset$ if $a \neq a^{\prime \prime}$ or $a^{\prime} \neq a^{\prime \prime \prime}$. A sequence of nodes $\left\{a_{0}, a_{1}, \ldots, a_{K}\right\}$ is a node-path from $a_{0}$ to $a_{K}$ if and only if for any $k=1, \ldots, K, \mathcal{R}\left(a_{k-1}, a_{k}\right) \neq \emptyset$. A transportation network $(A, \mathcal{G})$ forms a node-tree if and only if for any ordered pair of nodes $\left(a, a^{\prime}\right)$, there is a unique node-path from $a$ to $a^{\prime}$ (see Figure 3 ). Note that the network in Example 1 does not have a node-tree since there are two node paths $\{a, b, d\}$ and $\{a, c, d\}$ from $a$ to $d$. Given that a transportation network forms a nodetree, for commuter $i \in I\left(a, a^{\prime}\right)$, the set of available paths $\Omega\left(a, a^{\prime}\right)$ can be denoted by $\Omega\left(a, a^{\prime}\right)=\Pi_{k=1}^{K} \mathcal{R}\left(a_{k-1}, a_{k}\right)$, where $\left\{a_{0}, \ldots, a_{K}\right\}$ is a node-path from $a=a_{0}$ to $a^{\prime}=a_{K}$ (if there is no noncommuting option). For commuter $i \in I\left(a, a^{\prime}\right)$, utility function $u_{i}$ is weakly separable if and only if we can write $u_{i}$ as a strictly increasing function of subutilities from segments of paths:

$$
u_{i}(\omega, m)=U_{i}\left(v_{i}\left(\rho_{1}, n_{\rho_{1}}(m)\right), \ldots, v_{i}\left(\rho_{K}, n_{\rho_{K}}(m)\right)\right)
$$

where $\omega=\left(\rho_{1}, \ldots, \rho_{K}\right) \in \Pi_{k=1}^{K} \mathcal{R}\left(a_{k-1}, a_{k}\right), U_{i}$ is strictly increasing in its arguments, and $v_{i}\left(\rho_{k}, n_{\rho_{k}}(m)\right)$ is commuter $i$ 's subutility function for the $k$-th segment of paths from choosing $\rho_{k} \in \mathcal{R}\left(a_{k-1}, a_{k}\right)$, which is strictly decreasing in the user population $n_{\rho_{k}}$ (NEC). Weak separability excludes complementarities across choices in different segments of paths. With complementarities, we can easily modify Example 1 to achieve nonuniqueness of user equilibrium in a node-tree network. Our positive result 
in this section is the following.

Theorem 4. Suppose that $($ i) $(A, \mathcal{G})$ forms a node-tree, (ii) utility functions are weakly separable, and (iii) there is no noncommuting option. Under AN, NS, and NEC, user equilibrium allocation is essentially unique.

Proof. The proof is a trivial application of that of Theorem 1. Under the listed assumptions, at each node $a \in A$, the set of commuters to go to any next node $a^{\prime} \in A$ with $\mathcal{R}\left(a, a^{\prime}\right) \neq \emptyset$ is predetermined (given inelastic demand plus the node-tree property). Thus we can apply Theorem 1 to this segment by weakly separable utility functions, and user equilibrium allocation at this segment is essentially unique. This argument applies to any pair of directly connected nodes, and user equilibrium must be essentially unique.

What about the case with a noncommuting option? Actually, we may have multiple user equilibria even when the transportation network has a node-tree. The next example is constructed using the same logic as in Example 1.

EXAmple 4 . There is a transportation network connecting four nodes $\{a, b, c, d\}$ with five links: $\rho_{1}(a \rightarrow b), \rho_{2}(b \rightarrow c), \rho_{3}(b \rightarrow c), \rho_{4}(c \rightarrow d), \rho_{5}(c \rightarrow d)$ (see Figure 4). There are three types of atomless commuters (types 1,2 , and 3 ) commuting from node $a$ to node $d$. The measures of types 1,2 , and 3 are 1.2, 1, and 1, respectively. There are four paths $\Omega=\left\{\omega_{1}, \omega_{2}, \omega_{3}, \omega_{4}\right\}$, where $\omega_{1}=\left(\rho_{1}, \rho_{2}, \rho_{4}\right), \omega_{2}=\left(\rho_{1}, \rho_{2}, \rho_{5}\right), \omega_{3}=$ 
$\left(\rho_{1}, \rho_{3}, \rho_{4}\right)$, and $\omega_{4}=\left(\rho_{1}, \rho_{3}, \rho_{5}\right)$. There are noncommuting options. Each commuter's utility function is additively separable over links: $\sum_{\rho \in \omega} v_{i}\left(\rho, n_{\rho}\right)$, unless $\omega=\emptyset$. The three types of commuters have the following subutility functions, respectively:

Type 1:

$$
\begin{aligned}
v_{1}\left(\rho_{1}, n\right) & =v_{1}\left(\rho_{2}, n\right)=v_{1}\left(\rho_{4}, n\right)=-n, \\
v_{1}\left(\rho_{3}, n\right) & =v_{1}\left(\rho_{5}, n\right)=-100-n, \\
u_{1}(\emptyset) & =-3.8
\end{aligned}
$$

Type 2:

$$
\begin{aligned}
v_{2}\left(\rho_{1}, n\right) & =v_{2}\left(\rho_{5}, n\right)=-n, \\
v_{2}\left(\rho_{2}, n\right) & =19-20 n, \\
v_{2}\left(\rho_{3}, n\right) & =v_{2}\left(\rho_{4}, n\right)=-100-n, \\
u_{2}(\emptyset) & =-5 ;
\end{aligned}
$$

Type 3:

$$
\begin{aligned}
v_{3}\left(\rho_{1}, n\right) & =v_{3}\left(\rho_{3}, n\right)=-n, \\
v_{3}\left(\rho_{4}, n\right) & =19-20 n, \\
v_{3}\left(\rho_{2}, n\right) & =v_{2}\left(\rho_{5}, n\right)=-100-n, \\
u_{3}(\emptyset) & =-5 .
\end{aligned}
$$


Example 4 possesses two distinct user equilibria in which everybody in the same type chooses the same path. In user equilibrium (A), type 1 commuters choose $\omega_{1}$ and types 2 and 3 choose not to commute $(\emptyset)$. In user equilibrium B, type 1 commuters choose not to commute $(\emptyset)$ and types 2 and 3 choose $\omega_{2}$ and $\omega_{3}$, respectively. This example shows that we cannot generalize Theorem 1, if commuters are allowed a noncommuting option.

What about efficiency? Note that Theorem 4 does not mention efficiency of user equilibrium. Unfortunately, Milchtaich (2001) demonstrates that the simplest possible node-tree may not achieve efficiency even if commuters are homogeneous and there is no noncommuting option. In the paper, he characterizes network topologies that generate inefficient user equilibrium. The following intriguing example is taken from the paper. Unlike Braess's example, there is no way to support an efficient allocation as a user equilibrium by closing links.

Example 5 (Milchtaich, 2001). There is a transportation network $(A, \mathcal{G})$ with $A=\{a, b, c\}$ and $\mathcal{G}=\left\{\rho_{1}, \rho_{2}, \rho_{3}, \rho_{4}\right\}, \rho_{1}(a \rightarrow b), \rho_{2}(a \rightarrow b), \rho_{3}(b \rightarrow c), \rho_{4}(b \rightarrow c)$ (see Figure 5). There are homogeneous commuters commuting from $a$ to $c$. The population measure is assumed to be unity. There are four paths $\Omega=\left\{\omega_{1}, \omega_{2}, \omega_{3}, \omega_{4}\right\}$, where $\omega_{1}=\left(\rho_{1}, \rho_{3}\right), \omega_{2}=\left(\rho_{1}, \rho_{4}\right), \omega_{3}=\left(\rho_{2}, \rho_{3}\right), \omega_{4}=\left(\rho_{2}, \rho_{4}\right)$. There is no noncommuting option. Each commuter has an identical utility function that is additively separable over links: $\sum_{\rho \in \omega} v\left(\rho, n_{\rho}\right)$. Commuters have the following subutility functions:

$$
v\left(\rho_{1}, n\right)=v\left(\rho_{3}\right)=-2 n
$$




$$
v\left(\rho_{2}, n\right)=v\left(\rho_{4}\right)=-n-2
$$

In the unique user equilibrium, all commuters choose path $\omega_{1}$, and they get utilities

-4 . However, if measure $\frac{1}{2}$ population choose $\omega_{2}$ and the other half choose $\omega_{3}$, then commuters' utilities are -3.5 .

\section{Bottleneck Models with Simple Networks}

The bottleneck model introduces the dimension of time to the model. It is assumed that only a limited amount of traffic can go through a bottleneck of a transportation path at a certain time period, and leftover traffic forms a queue that generates a time delay in arriving at destinations. It is true that we can generalize the basic model to accommodate bottleneck models by renaming $\omega$ as a pair of paths and times $(\omega, t)$. However, the bottleneck model does not satisfy NS, since the length of a queue formed by commuters who choose $t^{\prime}<t$ affects the utility of a commuter who chooses to depart at $t$. Moreover, NEC is not satisfied in a strict sense. Thus we cannot apply our theorems directly to the bottleneck model even if the network is simple. In this section, we extend our Theorem 1 to such cases.

There are many papers that investigate user equilibrium in bottleneck models. Smith (1984) shows existence of user equilibrium in a model with a single bottleneck (with a single path) when schedule delay cost functions are common across consumers. Daganzo (1985) proves uniqueness of user equilibrium under essentially the same condition. Newell (1987) extends the analysis in the case of heterogeneous schedule delay functions, although they are assumed to be linear. An intertemporal model 
that analyzes mass-transit in Kraus and Yoshida (2002) is also related to Newell (1987). Wie (1995) extends single path models to a simple network model with a bottleneck for each path. He characterizes the equilibrium by using a differential game approach. Richter, Griffin, and Arnott (1992) analyze the same model by using a convex programming approach. Although many papers listed above (except for Richter, Griffin, and Arnott, 1992) assume continuous time, we employ a discrete time model in order to apply a similar argument in the previous sections. Unlike other papers, we allow commuter preferences to be very general.

Let $\{1, \ldots, T\}$ be the set of timings, where a smaller index means an earlier time. Each commuter chooses her commuting plan $(\omega, t) \in \Omega \times\{1, \ldots, T\} \cup\{\emptyset\}$, which consists of a path and a time. For notational convenience, we let $\left(\omega_{\emptyset}, t_{\emptyset}\right)=\emptyset$. We assume that the transportation network $\mathcal{G}$ is simple. The number of commuters who choose $(\omega, t)$ is denoted by $m_{\omega t}$, and $m=\left(m_{\omega t}\right)_{(\omega, t) \in \Omega \times\{1, \ldots, T\} \cup\{\emptyset\}}$. The capacity of a path $\omega$ at $t$ is denoted by $c_{\omega t}$, and the length of a queue of path $\omega$ at period $t$ (formed by commuters who chose to depart at time $t^{\prime} \leq t$ ) is denoted by $q_{\omega t}(m)$, where $q_{\omega 1}(m)=\max \left\{0, m_{\omega 1}-c_{\omega 1}\right\}$, and for any $t=2, \ldots, T, q_{\omega t}(m)$ is recursively defined by $q_{\omega t}(m)=\max \left\{0, q_{\omega t-1}(m)+m_{\omega t}-c_{\omega t}\right\}$. Thus this is a first-in-first-out (FIFO) model. Note that $q_{\omega t}(m)$ is not affected by $\left(m_{\omega t+1}, \ldots, m_{\omega T}\right)$. Each commuter's utility is a function of her commuting plan $(\omega, t)$ and the length of queue at $(\omega, t)$ : $u=u\left(\omega, t, q_{\omega t}(m)\right)$. Queue $q_{\omega t}(m)=0$ means that there is no congestion at $(\omega, t)$. The utility function is strictly decreasing in the length of queue $q$ at any commuting plan $(\omega, t)$. As usual, if commuter $i$ chooses $\emptyset=\left(\omega_{\emptyset}, t_{\emptyset}\right)$, then she can secure her utility at $u_{i}(\emptyset)$. We call this model a bottleneck model with a simple network. 
We can extend the proof of Theorem 1 to this case.

Theorem 5. In a bottleneck model with a simple network, user equilibrium allocation is essentially unique if $\mu\left(\left\{i \in I: u_{i}(\omega, t, 0)=u_{i}\left(\omega^{\prime}, t^{\prime}, 0\right)\right\}\right)=0$ for any $(\omega, t),\left(\omega^{\prime}, t^{\prime}\right) \in$ $\Omega \times\{1, \ldots, T\} \cup\{\emptyset\}$ with $(\omega, t) \neq\left(\omega^{\prime}, t^{\prime}\right)$.

Proof. Let $f$ and $g$ be equilibria with $m(f) \neq m(g)$. Then there exists $\omega_{0} \in \Omega$ with $m_{\omega_{0}}(f) \neq m_{\omega_{0}}(g)$. Let $t_{0} \in\{1, \ldots, T\}$ such that $m_{\omega_{0} t_{0}}(f) \neq m_{\omega_{0} t_{0}}(g)$ yet $m_{\omega_{0} t}(f)=$ $m_{\omega_{0} t}(g)$ for any $t<t_{0}$. Without loss of generality, assume $m_{\omega_{0} t_{0}}(f)<m_{\omega_{0} t_{0}}(g)$. Since we have $q_{\omega_{0} t_{0}-1}(m(f))=q_{\omega_{0} t_{0}-1}(m(g))\left(\right.$ unless $\left.t_{0}=1\right), q_{\omega_{0} t_{0}}(m(f)) \leq q_{\omega_{0} t_{0}}(m(g))$ must follow. Since $\Omega \times\{1, \ldots, T\}$ is finite, there is a subset $C_{0} \subset I$ with $\mu\left(C_{0}\right)>0$ and $\left(\omega_{1}, t_{1}\right)=f(i) \neq g(i)=\left(\omega_{0}, t_{0}\right)$ for $i$ a.e. in $C_{0}$. (Note that $f(i)=\emptyset$ for $i$ a.e. in $C_{0}$ cannot happen, since $\mu\left(\left\{i \in I: u_{i}\left(\omega_{0}, t_{0}, 0\right)=u_{i}(\emptyset)\right\}\right)=0$ and $u_{i}(\omega, t, n)$ is strictly increasing in $n$.) Since commuters in $C_{0}$ could have chosen $\left(\omega_{0}, t_{0}\right)$ under allocation $m(f)$, we have $u_{i}\left(\omega_{0}, t_{0}, q_{\omega_{0} t_{0}}(m(f))\right) \leq u_{i}\left(\omega_{1}, t_{1}, q_{\omega_{1} t_{1}}(m(f))\right)$ for $i$ a.e. in $C_{0}$. Since $q_{\omega_{0} t_{0}}(m(f)) \leq q_{\omega_{0} t_{0}}(m(g))$, we have $u_{i}\left(\omega_{0}, t_{0}, q_{\omega_{0} t_{0}}(m(g))\right) \leq u_{i}\left(\omega_{0}, t_{0}, q_{\omega_{0} t_{0}}(m(f))\right)$ a.e. in $I$. Since $g$ is a user equilibrium, we have $u_{i}\left(\omega_{1}, t_{1}, q_{\omega_{1} t_{1}}(m(g))\right) \leq u_{i}\left(\omega_{0}, t_{0}, q_{\omega_{0} t_{0}}(m(g))\right)$.

We now show $q_{\omega_{1} t_{1}}(m(g))>q_{\omega_{1} t_{1}}(m(f))$. To prove this, it is sufficient to show that $u_{i}\left(\omega_{1}, t_{1}, q_{\omega_{1} t_{1}}(m(g))\right)<u_{i}\left(\omega_{1}, t_{1}, q_{\omega_{1} t_{1}}(m(f))\right)$ for $i$ a.e. in $C_{0}$. First suppose that $u_{i}\left(\omega_{0}, t_{0}, q_{\omega_{0} t_{0}}(m(g))\right)=u_{i}\left(\omega_{0}, t_{0}, q_{\omega_{0} t_{0}}(m(f))\right)$ a.e. in $I$. Then, have $q_{\omega_{0} t_{0}}(m(g))=$ $q_{\omega_{0} t_{0}}(m(f))=0$. Since we assume that $u_{i}\left(\omega_{0}, t_{0}, 0\right)=u_{i}\left(\omega_{1}, t_{1}, 0\right)$ holds only for measure zero commuters, $u_{i}\left(\omega_{0}, t_{0}, q_{\omega_{0} t_{0}}(m(f))\right)<u_{i}\left(\omega_{1}, t_{1}, q_{\omega_{1} t_{1}}(m(f))\right)$ for $i$ a.e. in $C_{0}$. Together with $u_{i}\left(\omega_{1}, t_{1}, q_{\omega_{1} t_{1}}(m(g))\right) \leq u_{i}\left(\omega_{0}, t_{0}, q_{\omega_{0} t_{0}}(m(g))\right)$, we can conclude $u_{i}\left(\omega_{1}, t_{1}, q_{\omega_{1} t_{1}}(m(g))\right)<u_{i}\left(\omega_{1}, t_{1}, q_{\omega_{1} t_{1}}(m(f))\right)$ for $i$ a.e. in $C_{0}$. Second, suppose 
that $u_{i}\left(\omega_{0}, t_{0}, q_{\omega_{0} t_{0}}(m(g))\right)<u_{i}\left(\omega_{0}, t_{0}, q_{\omega_{0} t_{0}}(m(f))\right)$ for $i$ a.e. in $I$. In this case, the desired inequality obviously follows.

Since $q_{\omega_{1} t_{1}}(m(g))>q_{\omega_{1} t_{1}}(m(f))$, there exists $t^{\prime} \leq t$ such that $m_{\omega_{1} t_{1}^{\prime}}(g)>m_{\omega_{1} t_{1}^{\prime}}(f)$ and $q_{\omega_{1} t_{1}^{\prime}}(m(g))>q_{\omega_{1} t_{1}^{\prime}}(m(f))$. Thus there is again a subset of commuters $C_{1}$ with $\mu\left(C_{1}\right)>0$ and $\left(\omega_{2}, t_{2}\right)=f(i) \neq g(i)=\left(\omega_{1}, t_{1}^{\prime}\right)$ for $i$ a.e. in $C_{1}$. Note that we can choose $\left(\omega_{2}, t_{2}\right) \neq\left(\omega_{0}, t_{0}\right)$. By the same argument, we can show $q_{\omega_{2} t_{2}}(m(g))>$ $q_{\omega_{2} t_{2}}(m(f))$. Hence there is a sequence $\left\{\left(\omega_{0}, t_{0}, t_{0}^{\prime}, C_{0}\right),\left(\omega_{1}, t_{1}, t_{1}^{\prime}, C_{1}\right), \ldots\right\}$ such that for each $k,\left(\omega_{k}, t_{k}^{\prime}\right)=g(i) \neq f(i)=\left(\omega_{k+1}, t_{k+1}\right)$ for $i$ a.e. in $C_{k}$, where $t_{k}^{\prime} \leq t_{k}\left(t_{0}^{\prime}=t_{0}\right.$ for $k=0)$. By construction, we can choose $\left(\omega_{k+1}, t_{k+1}\right) \in \Omega \times\{1, \ldots, T\} \backslash \cup_{l=1}^{k}\left(\omega_{l}, t_{l}\right)$ for each $k$. However, both $\Omega$ and $T$ are finite. This is a contradiction. Hence user equilibrium is essentially unique.

Note that the above FIFO setting has some inconsistency in the model due to finiteness of $T$. Suppose that at the end of $(\omega, t-1)$, there is a queue with length $q_{\omega t-1}(m)$. Then, if in period $t, m_{\omega t}>c_{\omega t}$ of commuters are arriving, then even if we assume FIFO, some of the newly arrived commuters would face a queue with length $q_{\omega t-1}(m)$, while some of them would face a queue with length $q_{\omega t-1}(m)+m_{\omega t}-c_{\omega t}$. Nonetheless, in the above model, we assume that everybody faces a queue with length $q_{\omega t-1}(m)+m_{\omega t}-c_{\omega t}$. Isn't this inconsistent? Strictly speaking, the answer is yes. However, if $T$ is large enough, $m_{\omega t}-c_{\omega t}$ becomes smaller, and this difference becomes negligible (as in a continuous time model). On the other hand, if we adopt an expected waiting time criterion in evaluating commuters' utility when $T$ is small, the result in Theorem 5 may be overturned even in the homogeneous commuter case. The following 
example illustrates this point.

Example 6. Suppose that $\Omega=\{\omega\}, T=2$, capacity $c$ is constant for each period $c=$ $\frac{2}{3}$, and a homogeneous commuter's utilities given her choice $t=1,2$ and distribution of other commuters' choices $m=\left(m_{1}, m_{2}\right) \in \Delta^{\{1,2\}}$ are as follows:

$$
\begin{aligned}
& U(1, m)=-\frac{1}{9} \quad \text { if } m_{1} \leq \frac{2}{3}, \\
& U(1, m)=-\frac{1}{9}-\int_{0}^{\frac{2}{3}} 0 \times d n-\int_{\frac{2}{3}}^{m_{1}}\left(n-\frac{2}{3}\right) d n \\
& =-\frac{1}{9}-\left[\frac{m_{1}^{2}}{2}-\frac{2}{3} m_{1}+\frac{2}{9}\right] \quad \text { if } m_{1}>\frac{2}{3} \text {. } \\
& U(2, m)=-\max \left\{m_{1}-\frac{2}{3}, 0\right\} \quad \text { if } m_{2} \leq \frac{2}{3}, \\
& U(2, m)=-\max \left\{m_{1}-\frac{2}{3}, 0\right\}-\int_{0}^{\frac{2}{3}} 0 \times d n-\int_{\frac{2}{3}}^{m_{2}}\left(n-\frac{2}{3}\right) d n \\
& =-\max \left\{m_{1}-\frac{2}{3}, 0\right\}-\left[\frac{m_{2}^{2}}{2}-\frac{2}{3} m_{2}+\frac{2}{9}\right] \quad \text { if } m_{2}>\frac{2}{3} \text {, }
\end{aligned}
$$

where $-\frac{1}{9}$ in $U(1, m)$ is a disutility to departing earlier, $-\int_{0}^{\frac{2}{3}} 0 \times d n-\int_{\frac{2}{3}}^{m_{t}}\left(n-\frac{2}{3}\right) d n$ is the expected queue length when a within-period flow congestion at period $t$ is positive (when $m_{t}$ exceeds $c=\frac{2}{3}$ ), and $-\max \left\{m_{1}-\frac{2}{3}, 0\right\}$ is an accumulated queue from period 1.

Example 6 has at least two equilibria, $m=\left(m_{1}, m_{2}\right)=(0,1)$ and $m^{\prime}=\left(m_{1}^{\prime}, m_{2}^{\prime}\right)=$ $(1,0)$. To verify this, first check allocation $m$. Commuters' utility levels are $-\left[\frac{1}{2}-\frac{2}{3}+\frac{2}{9}\right]=$ $-\frac{1}{18}$. If a commuter deviates from this, she gets $-\frac{1}{9}$, which does not pay. Now consider $m^{\prime}$. Their utility levels are $-\frac{1}{9}-\frac{1}{18}=-\frac{1}{6}$. If a commuter deviates from this, she gets 
$-\frac{1}{3}$, which again does not pay. Thus there are two equilibria that are Pareto-ordered. Essentially, these multiple equilibria are the outcome of a coordination problem due to the difference between the expected cost of waiting given a within-period flow congestion $\left(-\frac{1}{18}\right)$ and the cost of waiting given an accumulated queue from the previous period $\left(-\frac{1}{3}\right)$. This discrepancy would be significant only when $T$ is small and the grid of period divisions is not fine enough.

\section{A Mysterious Coincidence: Existence in Finite Models and Uniqueness in Continuum Models}

There is an intriguing relationship between the nonemptiness of user equilibrium in finite models (models with a finite number of commuters) and the uniqueness of user equilibrium in continuum models. Somewhat surprisingly, the conditions that are required to guarantee the existence of user equilibrium in a finite model are very similar to the ones that are required to guarantee the uniqueness of user equilibrium in continuum models. We discuss these two cases in order.

\subsection{Simple Networks with General Utility Functions}

When there is a continuum of atomless commuters, we know, from Theorem 1 , that AN, NS, and NEC suffice to guarantee uniqueness of user equilibrium if the network is simple, and we also know from Example 1 that there may be multiple user equilibria, otherwise. Now, let us consider a finite commuter problem: a finite commuter problem is a list $\left(I, A, \mathcal{G}, \bar{\Omega},\left(u_{i}\right)_{i \in I}\right)$, where $I=\{1, \ldots,|I|\}$ is a finite set of commuters and $u_{i}=\left(\left(u_{i}(\omega, \cdot)\right)_{\omega \in \Omega}, u_{i}(\emptyset)\right)$ is commuter $i$ 's utility function, where $u_{i}(\omega, \cdot): Z_{++} \rightarrow$ 
$\Re$ is commuter $i$ 's utility function when she chooses path $\omega$. An allocation is $f: I \rightarrow \bar{\Omega}$ where $f(i) \in \bar{\Omega}$ for any $i \in I$. The collection of all feasible allocations is denoted by $F$. A pure strategy user equilibrium in an anonymous finite model is an allocation $f$ such that $u_{i}(f(i), m(f)) \geq u_{i}\left(\omega, m\left(f^{(i, \omega)}\right)\right)$ for any $\omega \in \bar{\Omega} \backslash\{f(i)\}$, where $f^{(i, \omega)}: I \rightarrow \bar{\Omega}$ with $f^{(i, \omega)}(j)=f(j)$ for any $j \neq i$ and $f^{(i, \omega)}(i)=\omega$. In the literature of game theory, Milchtaich (1996), Konishi, Le Breton, and Weber (1997a), and Quint and Shubik (1994) analyze finite "congestion games" with simple networks (in our context). They prove that under the exactly the same conditions (AN, NS, and NEC: adjusting these definitions to finite problem versions is straightforward), there exists a user equilibrium if the network is simple. Note that the proofs are completely different from each other. Milchtaich (2000) uses a limit approach to analyze the sets of user equilibria of congestion games in the finite and continuum cases. Consider a sequence of finite games generated by duplicating a finite game repeatedly. He finds that the sequence of the sets of equilibria in these games shrinks to a singleton. The unique element in the limit set is the unique equilibrium of the limit infinite game. However, it is still a mystery how the same network restriction obtains existence in finite games and uniqueness in continuum games. In the following, we provide an example of a finite problem, which has no user equilibrium (in pure strategies) when the network is not simple.

Example 7. There is a transportation network connecting five nodes $\{a, b, c, d, e\}$ with eight links $\left\{\rho_{1}, \rho_{2}, \ldots, \rho_{8}\right\}$, where $\rho_{1}(a \rightarrow b), \rho_{2}(b \rightarrow e), \rho_{3}(b \rightarrow c), \rho_{4}(a \rightarrow c)$, $\rho_{5}(c \rightarrow e), \rho_{6}(d \rightarrow c), \rho_{7}(a \rightarrow d)$, and $\rho_{8}(d \rightarrow e)$ (see Figure 6-1). There are three commuters commuting from node $a$ to node $e$. The set of commuters is $=\{1,2,3\}$. 
There are five paths $\Omega=\left\{\omega_{1}, \omega_{2}, \ldots, \omega_{5}\right\}$, where $\omega_{1}=\left(\rho_{1}, \rho_{2}\right), \omega_{2}=\left(\rho_{1}, \rho_{3}, \rho_{5}\right)$, $\omega_{3}=\left(\rho_{4}, \rho_{5}\right), \omega_{4}=\left(\rho_{7}, \rho_{6}, \rho_{5}\right)$, and $\omega_{5}=\left(\rho_{7}, \rho_{8}\right)$. There is no noncommuting option. Each commuter's utility function is additively separable over links: $\sum_{\rho \in \omega} v_{i}\left(\omega, n_{\rho}\right)$. Three commuters' utility functions satisfy the following conditions on top of AN, NS, and NEC:

$$
\begin{aligned}
v_{1}\left(\rho_{3}, 1\right)+v_{1}\left(\rho_{5}, 3\right) & >v_{1}\left(\rho_{2}, 1\right)>v_{1}\left(\rho_{3}, 2\right)+v_{1}\left(\rho_{5}, 2\right)>v_{1}\left(\rho_{3}, 2\right)+v_{1}\left(\rho_{5}, 3\right), \\
v_{1}\left(\rho_{4}, 1\right) & =v_{1}\left(\rho_{7}, 1\right)=-L ; \\
v_{2}\left(\rho_{4}, 1\right)+v_{2}\left(\rho_{5}, 2\right) & >v_{2}\left(\rho_{7}, 1\right)+v_{2}\left(\rho_{8}, 1\right)>v_{2}\left(\rho_{7}, 2\right)+v_{2}\left(\rho_{8}, 1\right)>v_{2}\left(\rho_{4}, 1\right)+v_{2}\left(\rho_{5}, 3\right), \\
v_{2}\left(\rho_{1}, 1\right) & =v_{2}\left(\rho_{6}, 1\right)=-L ; \\
v_{3}\left(\rho_{7}, 1\right)+v_{3}\left(\rho_{6}, 1\right) & >v_{3}\left(\rho_{1}, 2\right)+v_{3}\left(\rho_{3}, 1\right)>v_{3}\left(\rho_{1}, 2\right)+v_{3}\left(\rho_{3}, 2\right)>v_{3}\left(\rho_{7}, 2\right)+v_{3}\left(\rho_{6}, 1\right), \\
v_{3}\left(\rho_{2}, 1\right) & =v_{3}\left(\rho_{4}, 1\right)=v_{3}\left(\rho_{8}, 1\right)=-L ;
\end{aligned}
$$

where $L$ is a sufficiently large number such that commuter $i$ would not use such a link under any situation.

Claim 3. Example 7 does not possess a pure strategy user equilibrium.

Proof. Observe that players 1,2 , and 3 choose from sets of paths $\left\{\omega_{1}, \omega_{2}\right\},\left\{\omega_{3}, \omega_{5}\right\}$, and $\left\{\omega_{2}, \omega_{4}\right\}$, respectively. Given this, there are eight strategy profiles (in pure strategies): $A=\left(\omega_{1}, \omega_{3}, \omega_{2}\right), B=\left(\omega_{1}, \omega_{3}, \omega_{4}\right), C=\left(\omega_{1}, \omega_{5}, \omega_{2}\right), D=\left(\omega_{1}, \omega_{5}, \omega_{4}\right)$, $E=\left(\omega_{2}, \omega_{3}, \omega_{2}\right), F=\left(\omega_{2}, \omega_{3}, \omega_{4}\right), G=\left(\omega_{2}, \omega_{5}, \omega_{2}\right)$, and $H=\left(\omega_{2}, \omega_{5}, \omega_{4}\right)$. As we can easily see, at each strategy profile a commuter changes her path: $A \rightarrow_{3} B$, $B \rightarrow_{1} F, C \rightarrow_{2} A, D \rightarrow_{3} C, E \rightarrow_{1} A, F \rightarrow_{2} H, G \rightarrow_{1} C$, and $H \rightarrow_{3} G$. For ex- 
ample, $A \rightarrow_{3} B$ is interpreted as follows: if the strategy profile is $A$, then commuter 3 switches her path, and this change results in a new strategy profile $B$ (see Figure 6-2). This example does not possess a pure strategy user equilibrium.

Example 7 describes the importance of a "simple" network in obtaining the existence of user equilibrium in finite models. It can be considered as a counterpart to Example 1. Example 1 shows the nonuniqueness of user equilibrium in a continuum model if the network is not simple.

\subsection{Daganzo's Restriction over Preferences}

We first show that Daganzo's (1983) assumptions can be applied to finite games. We can prove this by using an idea in Konishi, Le Breton, and Weber (1997b) to extend Rosenthal's (1973) potential function approach. The proof of the theorem is found in the appendix. The potential function approach has been used in both continuum and finite models (Beckmann et. al. (1956) for continuum models, and Rosenthal (1973) for finite models). In the appendix, we briefly discuss how this approach loosely links these two different problems under Daganzo's preference restriction.

Theorem 6. Consider a finite commuter problem in which $u_{i}(\omega, m)=\sum_{\rho \in \omega}\left(\theta_{\rho i}-c_{\rho}\left(n_{\rho}(m)\right)\right)$ for any $i \in I$. This problem has a pure strategy user equilibrium.

Without Daganzo's assumption, a finite commuter problem may not have a pure strategy user equilibrium. This can be seen from Example 7 again. The following claim shows that preferences in Example 7 violate Daganzo's assumption.

Claim 4. Example 7 violates Daganzo's preference restriction. 
Proof. From the inequalities over $v_{i} \mathrm{~s}$, we obtain:

$$
\begin{aligned}
& v_{1}\left(\rho_{3}, 1\right)-v_{1}\left(\rho_{3}, 2\right)>v_{1}\left(\rho_{5}, 2\right)-v_{1}\left(\rho_{5}, 3\right), \\
& v_{2}\left(\rho_{5}, 2\right)-v_{2}\left(\rho_{5}, 3\right)>v_{2}\left(\rho_{7}, 1\right)-v_{2}\left(\rho_{7}, 2\right), \\
& v_{3}\left(\rho_{7}, 1\right)-v_{3}\left(\rho_{7}, 2\right)>v_{3}\left(\rho_{3}, 1\right)-v_{3}\left(\rho_{3}, 2\right) .
\end{aligned}
$$

Suppose that Daganzo's preference restriction is satisfied. Then, these three inequalities imply

$$
\begin{aligned}
& c_{\rho_{3}}(1)-c_{\rho_{3}}(2)>c_{\rho_{5}}(2)-c_{\rho_{5}}(3), \\
& c_{\rho_{5}}(2)-c_{\rho_{5}}(3)>c_{\rho_{7}}(1)-c_{\rho_{7}}(2), \\
& c_{\rho_{7}}(1)-c_{\rho_{7}}(2)>c_{\rho_{3}}(1)-c_{\rho_{3}}(2) .
\end{aligned}
$$

This is a contradiction. Thus Daganzo's preference restriction is not satisfied in Example 7.

\section{Concluding Remarks}

In this paper, we provide sufficient conditions on the shape of transportation networks that guarantee the uniqueness of user equilibrium when there are heterogeneous commuters. We impose three conditions: anonymity, no spillovers, and negative externality from congestion. Under these reasonable conditions, we show that as long as a transportation network is simple, then user equilibrium is essentially unique in general. However, once we depart from simple networks, we need to impose further 
qualifications to guarantee uniqueness. One may question if anonymous congestion is a good approximation of the real world. Of course, it might not be. Bad drivers may slow down traffic more than good drivers. However, using Daganzo's (1983) technique, we can easily translate this situation to our anonymous framework in the following simple case: good drivers and bad drivers are perfect substitutes for each commuter's perception. Let $m^{b}(f)$ and $m^{g}(f)$ be bad and good drivers' distributions over paths. If bad drivers are always $t$ times more annoying to every commuter than good drivers, then effectively the level of congestion at path $\omega$ is $t m_{\omega}^{b}(f)+m_{\omega}^{g}(f)$. By defining $z_{\omega}(f) \equiv t m_{\omega}^{b}(f)+m_{\omega}^{g}(f)$, we can use exactly the same analysis in the paper. The equilibrium condition will not be affected because each commuter is atomless. Of course, if there is no such linear relationship, then we cannot apply this argument. However, it is easy to see that user equilibrium may not be unique, if congestion externalities across commuters are nonlinear.

\section{Appendix}

In this appendix, we first provide a proof of Daganzo's theorem. We then provide a proof of Theorem 6 and discuss a potential function approach.

Proof of Daganzo's Theorem (Daganzo, 1983). We will work on paths instead of links. Let $C_{\omega}(m) \equiv \sum_{\rho \in \omega} c_{\rho}\left(n_{\rho}(m)\right)$ and let $C \equiv\left(C_{\omega}\right)_{\omega \in \Omega}$. Let $\Theta_{\omega} \equiv \sum_{\rho \in \omega} \theta_{\omega}$ and $\Theta \equiv\left(\Theta_{\omega}\right)_{\omega \in \bar{\Omega}}$. In this special case, the space of utility functions is $\mathcal{U}=\Re^{\bar{\Omega}}$. We first derive aggregated demand correspondence. This correspondence describes 
how many commuters are willing to choose each path under congestion cost of $\omega$. Let $B\left(\omega, \omega^{\prime} ; C\right) \equiv\left\{\Theta \in \Re^{\bar{\Omega}}: \Theta_{\omega}-C_{\omega} \geq \Theta_{\omega^{\prime}}-C_{\omega^{\prime}}\right\}$, which denotes the set of commuter characteristics that weakly prefer $\omega$ to $\omega^{\prime}$ under $C$. Let $B_{\omega}^{*}(C) \equiv$ $\cap_{\omega^{\prime} \in \bar{\Omega} \backslash\{\omega\}} B\left(\omega, \omega^{\prime} ; C\right)$, which denotes the set of commuter characteristics that weakly prefer $\omega \in \bar{\Omega}$ to any other path including a noncommuting option. Given this, consumer's best response correspondence (demand correspondence) can be written as $\psi: \Re^{\bar{\Omega}} \times \Re_{+}^{\Omega} \rightarrow \bar{\Omega}$ such that $\psi(\Theta, C)=\left\{\omega \in \bar{\Omega}: \Theta \in B_{\omega}^{*}(C)\right\}$. Let $D: \Re_{+}^{\Omega} \rightarrow M$ be the aggregated (average) demand correspondence such that $D(C) \equiv \int_{\Theta} \psi(\Theta, C) d \mu$ (for an integration of a correspondence, see, e.g., Hildenbrand, 1974). The $\omega$ 's argument of $D(C)$ is denoted by $D_{\omega}(C)$ : the aggregate demand for path $\omega$ under $C$.

It is well known that a strictly increasing cost function $C: M \rightarrow \Re_{+}^{\Omega}$ satisfies "strict monotonicity": i.e., $\left(C\left(m^{\prime}\right)-C(m)\right)\left(m^{\prime}-m\right)>0$ for any $m, m^{\prime} \in M$ with $m \neq m^{\prime}$ and $C(m) \neq C\left(m^{\prime}\right)$ (see, e.g., Nagurney, 1993). Let $\mathcal{C}=\left\{C \in \Re_{+}^{\Omega}: C=C(m)\right.$ for some $m \in M\}$, and let $S: \mathcal{C} \rightarrow M$ be such that $S(C)=\{m \in M: C(m)=C\}$. Note that $\left(s^{\prime}-s\right)\left(C^{\prime}-C\right)>0$ also holds for any $C, C^{\prime} \in \mathcal{C}$ with $C^{\prime} \neq C$, and any $s^{\prime} \in S\left(C^{\prime}\right)$ and $s \in S(C)$.

We now describe equilibrium congestion level $C$. The user equilibrium congestion level $C^{*}$ can be described by a system of excess demand equations:

$$
Z\left(C^{*}\right) \equiv D\left(C^{*}\right)-S\left(C^{*}\right) \ni 0
$$

Thus we can show the uniqueness of solution $C^{*} \in \mathcal{C}$ of the above, if $\left(z^{\prime}-z\right)\left(C^{\prime}-C\right)<0$ (strong monotonicity) holds for any $C, C^{\prime} \in \mathcal{C}$ with $C^{\prime} \neq C$, and any $z^{\prime} \in Z\left(C^{\prime}\right)$ and 
$z \in Z(C)$. Note $z^{\prime}=d^{\prime}-s^{\prime}$ and $z=d-s$ for some $s^{\prime} \in S\left(C^{\prime}\right), s \in S(C), d^{\prime} \in D\left(C^{\prime}\right)$ and $d \in D(C)$. Thus what is left to show is that $\left(d^{\prime}-d\right)\left(C^{\prime}-C\right) \leq 0$ holds for any $C, C^{\prime} \in \mathcal{C}$ with $C^{\prime} \neq C$, and any $d^{\prime} \in D\left(C^{\prime}\right)$ and any $d \in D(C)$.

So, we prove this statement. Fix $C, C^{\prime}$, and pick any $d \in D(C)$ and any $d^{\prime} \in$ $D\left(C^{\prime}\right)$. Let $\Delta C \equiv C^{\prime}-C$ and $\Delta d \equiv d^{\prime}-d$. Reorder the elements of $\Omega$ according to the values of $\Delta C_{\omega}: \Delta C_{\omega_{1}} \geq \Delta C_{\omega_{2}} \geq \ldots \geq \Delta C_{\omega_{|\Omega|}}$. Note $B\left(\omega_{k}, \omega_{k^{\prime}} ; C\right) \equiv\left\{\Theta \in \Re^{\bar{\Omega}}\right.$ : $\left.\Theta_{\omega_{k}}-C_{\omega_{k}} \geq \Theta_{\omega_{k^{\prime}}}-C_{\omega_{k^{\prime}}}\right\}=\left\{\Theta \in \Re^{\bar{\Omega}}: \Theta_{\omega_{k}}-\Theta_{\omega_{k^{\prime}}} \geq C_{\omega_{k}}-C_{\omega_{k^{\prime}}}\right\}$. This implies

$$
B\left(\omega_{k}, \omega_{k^{\prime}} ; C+\Delta C\right) \subset B\left(\omega_{k}, \omega_{k^{\prime}} ; C\right)
$$

for any $k<k^{\prime}$, since $\left(C_{\omega_{k}}+\Delta C_{\omega_{k}}\right)-\left(C_{\omega_{k^{\prime}}}+\Delta C_{\omega_{k^{\prime}}}\right) \geq C_{\omega_{k}}-C_{\omega_{k^{\prime}}}$. Now define $\left\{\omega_{1}, \ldots, \omega_{k_{+}}\right\} \equiv\left\{\omega \in \Omega: \Delta C_{\omega}>0\right\}$ and $\left\{\omega_{k_{-}}, \ldots, \omega_{|\Omega|}\right\} \equiv\left\{\omega \in \Omega: \Delta C_{\omega}<0\right\}$; i.e., $\omega_{k_{+}}$is the path that has the smallest cost increase, and $\omega_{k_{-}}$is the path that has the smallest cost decrease. Since $C_{\emptyset}=0$, we have

$$
B\left(\omega_{k}, \emptyset ; C+\Delta C\right) \subset B\left(\omega_{k}, \emptyset ; C\right)
$$

for any $k \leq k_{+}$, and

$$
B\left(\omega_{k}, \emptyset ; C+\Delta C\right) \supset B\left(\omega_{k}, \emptyset ; C\right)
$$

for any $k \geq k_{-}$. Given these three inclusion relationships, we have $\cup_{l=1}^{k} B_{\omega_{l}}^{*}(C+$ $\Delta C) \subset \cup_{l=1}^{k} B_{\omega_{l}}^{*}(C)$ for any $k \leq k_{+}$, and $\cup_{l=k}^{|\Omega|} B_{\omega_{l}}^{*}(C+\Delta C) \supset \cup_{l=k}^{|\Omega|} B_{\omega_{l}}^{*}(C)$ for any $k \geq k_{-}$. This implies that we have $\sum_{l=1}^{k} d_{\omega_{l}}^{\prime} \leq \sum_{l=1}^{k} d_{\omega_{l}}$ for any $k \leq k_{+}$, and 
$\sum_{l=k}^{|\Omega|} d_{\omega_{l}}^{\prime} \geq \sum_{l=k}^{|\Omega|} d_{\omega_{l}}$ for any $k \geq k_{-} ;$or

$$
\sum_{l=1}^{k} \Delta d_{\omega_{l}} \leq 0 \text { for any } k \leq k_{+} \text {, and } \sum_{l=k}^{|\Omega|} \Delta d_{\omega_{l}} \geq 0 \text { for any } k \geq k_{-} .
$$

Then, we have (for any $d^{\prime} \in D(C+\Delta C)$ and $d \in D(C)$ ):

$$
\begin{aligned}
\sum_{l=1}^{k_{+}} \Delta d_{\omega_{l}} \Delta C_{\omega_{l}} & =\Delta d_{\omega_{1}} \Delta C_{\omega_{1}}+\Delta d_{\omega_{2}} \Delta C_{\omega_{2}}+\sum_{l=3}^{k_{+}} \Delta d_{\omega_{l}} \Delta C_{\omega_{l}} \\
& =\Delta d_{\omega_{1}}\left(\Delta C_{\omega_{1}}-\Delta C_{\omega_{2}}\right)+\Delta d_{\omega_{1}} \Delta C_{\omega_{2}}+\Delta d_{\omega_{2}} \Delta C_{\omega_{2}}+\sum_{l=3}^{k_{+}} \Delta d_{\omega_{l}} \Delta C_{\omega_{l}} \\
& \leq\left(\Delta d_{\omega_{1}}+\Delta d_{\omega_{2}}\right) \Delta C_{\omega_{2}}+\sum_{l=3}^{k_{+}} \Delta d_{\omega_{l}} \Delta C_{\omega_{l}} \\
& \leq\left(\Delta d_{\omega_{1}}+\Delta d_{\omega_{2}}+\Delta d_{\omega_{3}}\right) \Delta C_{\omega_{3}}+\sum_{l=4}^{k_{+}} \Delta d_{\omega_{l}} \Delta C_{\omega_{l}} \\
& \leq \ldots \leq\left(\sum_{l=1}^{k_{+}} \Delta d_{\omega_{l}}\right) \Delta C_{\omega_{k_{+}}} \leq 0 .
\end{aligned}
$$

The last inequality applies since $\Delta C_{\omega_{k_{+}}}>0$. Similarly, we have:

$$
\sum_{l=k_{-}}^{|\Omega|} \Delta d_{\omega_{l}} \Delta C_{\omega_{l}} \leq\left(\sum_{l=k_{-}}^{|\Omega|} \Delta d_{\omega_{l}}\right) \Delta C_{\omega_{k_{-}}} \leq 0
$$

Since $\Delta C_{\omega_{l}}=0$ for any $l$ with $k_{+}<l<k_{-}$, we obtain:

$$
\begin{aligned}
\left(d^{\prime}-d\right)\left(C^{\prime}-C\right) & =\sum_{\omega \in \Omega} \Delta d_{\omega} \Delta C_{\omega} \\
& =\sum_{l=1}^{k_{+}} \Delta d_{\omega_{l}} \Delta C_{\omega_{l}}+\sum_{l=k_{-}}^{|\Omega|} \Delta d_{\omega_{l}} \Delta C_{\omega_{l}}
\end{aligned}
$$




$$
\leq\left(\sum_{l=1}^{k_{+}} \Delta d_{\omega_{l}}\right) \Delta C_{\omega_{k_{+}}}+\left(\sum_{l=k_{-}}^{|\Omega|} \Delta d_{\omega_{l}}\right) \Delta C_{\omega_{k_{-}}} \leq 0
$$

The proof is complete.

Proof of Theorem 6. Without loss of generality, we normalize $u_{\emptyset}=0$. Consider a function $\Phi: F \rightarrow \Re$ such that

$$
\Phi(f) \equiv \sum_{i \in I}\left(\sum_{\rho \in f(i)} \theta_{\rho i}\right)-\sum_{\rho \in \mathcal{G}}\left(\sum_{k=1}^{\bar{n}_{\rho}(f)} c_{\rho}(k)\right),
$$

where $\bar{n}_{\rho}(f)=\sum_{\omega \ni \rho} m_{\omega}(f)$ is the number of commuters who use link $\rho$ under allocation $f$. Let $f^{*} \in \arg \max _{f \in F} \Phi(f)$. Since $F$ is a finite set, $f^{*}$ exists. We claim $f^{*}$ is a pure strategy user equilibrium. Suppose not. Then, there exist a commuter $i$ and a path $\omega \in \bar{\Omega} \backslash\left\{f^{*}(i)\right\}$ such that

$$
\sum_{\rho \in \omega} \theta_{\rho i}-\sum_{\rho \in \omega} c_{\rho}\left(\bar{n}_{\rho}\left(f^{*(i, \omega)}\right)\right)>\sum_{\rho \in f^{*}(i)} \theta_{\rho i}-\sum_{\rho \in f^{*}(i)} c_{\rho}\left(\bar{n}_{\rho}\left(f^{*}\right)\right)
$$

Note that $\bar{n}_{\rho}\left(f^{*(i, \omega)}\right)=\bar{n}_{\rho}\left(f^{*}\right)$ iff $\rho \notin \omega \cup f^{*}(i)$ or $\rho \in \omega \cap f^{*}(i), \bar{n}_{\rho}\left(f^{*(i, \omega)}\right)=\bar{n}_{\rho}\left(f^{*}\right)+1$ iff $\rho \in \omega \backslash f^{*}(i)$, and $\bar{n}_{\rho}\left(f^{*}\right)-1$ iff $\rho \in f^{*}(i) \backslash \omega$. Given this, it is easy to see the following:

$$
\begin{aligned}
\Phi\left(f^{*(i, \omega)}\right) & =\sum_{i \in I}\left(\sum_{\rho \in f^{*(i, \omega)}(i)} \theta_{\rho i}\right)-\sum_{\rho \in \mathcal{G}}\left(\sum_{k=1}^{\bar{n}_{\rho}\left(f^{*(i, \omega)}\right)} c_{\rho}(k)\right) \\
& =\Phi\left(f^{*}\right)+\sum_{\rho \in \omega} \theta_{\rho i}-\sum_{\rho \in f^{*}(i)} \theta_{\rho i}-\sum_{\rho \in \omega} c_{\rho}\left(\bar{n}_{\rho}\left(f^{*(i, \omega)}\right)\right)+\sum_{\rho \in f^{*}(i)} c_{\rho}\left(\bar{n}_{\rho}\left(f^{*}\right)\right) \\
& =\Phi\left(f^{*}\right)+\left(\sum_{\rho \in \omega} \theta_{\rho i}-\sum_{\rho \in \omega} c_{\rho}\left(\bar{n}_{\rho}\left(f^{*(i, \omega)}\right)\right)\right)-\left(\sum_{\rho \in f^{*}(i)} \theta_{\rho i}-\sum_{\rho \in f^{*}(i)} c_{\rho}\left(\bar{n}_{\rho}\left(f^{*}\right)\right)\right) \\
& >\Phi\left(f^{*}\right) .
\end{aligned}
$$


This contradicts the definition of $f^{*}$, and the proof is complete.

Function $\Phi$ in the proof of Theorem 6 is called a potential function (Rosenthal, 1973). Note that $\sum_{\rho \in f(i)} \theta_{\rho i}$ denotes the gross utility that commuter $i$ can obtain by choosing $f(i)$, and the content of the first parenthesis is the sum of gross utilities in the economy. However, the content of the second parenthesis is not the total cost in the economy. It is the sum of marginal costs when one by one commuters joined in this link. This theorem shows that Daganzo's preference restriction also guarantees the existence of a pure strategy user equilibrium in finite commuter problems. Again, note that the proof of Theorem 6 and the proof of Daganzo's theorem provided in the appendix are very different. Note also that there are differences in the conditions of the theorems if we generalize these two theorems. The proof of Daganzo's theorem allows more general cost functions as long as they exhibit strict monotonicity, while it requires NEC. In contrast, the proof of Theorem 6 works even if NEC is dropped, while it requires separability of cost functions over links.

We now briefly illustrate an alternative proof for the Daganzo theorem by using a potential function to extend Beckmann et al.'s (1956) analysis. This alternative proof requires separable cost functions. The discussion is based on a private correspondence with Igal Milchtaich. Following Mas-Colell (1984), we will use so-called distribution approach instead of working on allocation $f$. Without loss of generality, we assume $\mu(I)=1$. Let $\nu$ be the probability distribution of commuters over utility space $\mathcal{U}$ (here, the space of $\theta \mathrm{s}: \theta=\left(\theta_{\rho}\right)_{\rho \in \mathcal{G}}$ ), generated from $e: I \rightarrow \mathcal{U}$ and $\mu$. We introduce some more notation: let $\mathcal{B}_{E}$ be the collection of Borel sets on a metric space $E$. We 
denote by $\mathcal{M}_{E}$ the space of all probability measures on $E$. If $\tau \in \mathcal{M}_{E \times F}$, we denote by $\tau_{E}$ the marginal distribution on $E$. An allocation in distribution is a probability measure $\tau \in \mathcal{M}_{\mathcal{U} \times \bar{\Omega}}$, where $\tau_{\mathcal{U}}=\nu$. A point $(\theta, \omega) \in \mathcal{U} \times \bar{\Omega}$ describes a commuter of type $\theta$ choosing path $\omega$. Let the collection of allocations be $\mathcal{T} \equiv\left\{\tau \in \mathcal{M}_{\mathcal{U} \times \bar{\Omega}}\right.$ : $\left.\tau_{\mathcal{U}}=\nu\right\}$. Given this setup, we can construct a potential function in the following way: $\Phi: \mathcal{M}_{\mathcal{U} \times \bar{\Omega}} \rightarrow \Re$ such that

$$
\Phi(\tau)=\sum_{\omega \in \bar{\Omega}} \int_{\mathcal{U}}\left(\sum_{\rho \in \omega} \theta_{\rho}\right) d \tau_{\omega}-\sum_{\rho \in \mathcal{G}}\left(\int_{k=0}^{n_{\rho}(\tau)} c_{\rho}(k) d k\right)
$$

where $\tau_{\omega}$ is marginal distribution on $\omega \in \bar{\Omega}$, and $n_{\rho}(\tau)=\sum_{\omega \ni \rho} \int_{\mathcal{U}} d \tau_{\omega}$. Since we assume $c(\rho, k)$ is strictly increasing in $k, \int_{k=0}^{n_{\rho}(\tau)} c_{\rho}(k) d k$ is strictly convex in $n_{\rho}(\tau)$. As a result, $\Phi(\tau)$ is a strictly concave function. Consider the following problem:

$$
\max _{\tau \in \mathcal{M}_{\mathcal{U} \times \bar{\Omega}}} \Phi(\tau)
$$

Since $\mathcal{M}_{\mathcal{U} \times \bar{\Omega}}$ is a convex set, there is a unique solution to the above problem. We can show that the solution of the above problem is the only allocation in distribution that satisfies user equilibrium condition. Hence we can see that the potential function links these two problems closely.

There are other interesting applications of potential functions in transportation science. Jehiel (1993) considers a traffic corridor with multiple transportation modes with a continuum of entry points and a single exit point. Commuters with common preferences are distributed over the entry points, and they all commute to the exit 
point. In this generalized model, Jehiel proves uniqueness of user equilibrium by employing the potential function approach. Sandholm (2001b) analyzes evolutionary implementability of an efficient toll system in the standard homogeneous commuter setting. He finds a supporting toll system of an efficient allocation by using a potential function directly. Sandholm (2001a) discusses the stability of Nash equilibria in general games with a continuum of homogeneous players employing potential functions.

\section{ACKNOWLEDGMENTS}

In memory of Bob Rosenthal. The author thanks many colleagues and friends for their warm encouragement. Michel Le Breton originally exposed him to the general topic of transportation network problems. Correspondences with Igal Milchtaich were invaluable. Detailed comments and suggestions from two anonymous referees of the journal improved the readability of the paper dramatically. He thanks Richard Arnott, Marcus Berliant, Bob Helsley, Hsueh-Ling Huynh, Marvin Kraus, John Nachbar, Tony Smith, Takashi Ui, Erik Verhoef, and the participants of seminars at Boston College, Washington University, Autonomous University of Barcelona, University of Aarhus, Kyoto University, and Kagawa University for their helpful comments and suggestions. This work is partially supported by a fellowship from the JSPS.

\section{References}


1. S.P. Anderson, A. de Palma, and J.-F. Thisse, Discrete Choice Theory of Product Differentiation, (MIT Press), Cambridge (1992).

2. R. Arnott, A. de Palma, and R. Lindsey, "Route Choice with Heterogenous Drivers and Group-Specific Congestion Costs," Regional Science and Urban Economics 22, 71-102 (1992).

3. R. Arnott, A. de Palma, and R. Lindsey, "A Structural Model of Peak-Period Congestion: A Traffic Bottleneck with Elastic Demand," American Economic Review 83, 161-179 (1993).

4. R. Arnott and A. Yan, "The Two Mode Problem: Second Best Pricing and Capacity," Review of Urban and Regional Development Studies 12, 170-199 (2000).

5. M. Beckmann, C.B. McGuire, and C.B. Winsten, Studies in the Economics of Transportation, (Yale University Press), New Haven (1956).

6. D. Braess, "Uber ein Paradoxon der Verkersplanung," Unternehmenforschung $12,258-268(1968)$.

7. S. Dafermos, "Traffic Equilibria and Variational Inequalities," Transportation Science 14, 42-54 (1980).

8. C.F. Daganzo, "Stochastic Network Equilibrium with Multiple Vehicle Types and Asymmetric, Indefinite Link Cost Jacobians," Transportation Science 17, 282-300 (1983).

9. C.F. Daganzo, "The Uniqueness of a Time-Dependent Equilibrium Distribution of Arrivals at a Single Bottleneck," Transportation Science 19, $29-37$ (1985). 
10. W. Hildenbrand, Core and Equilibria of a Large Economy, (Princeton University Press), Princeton (1974).

11. P. Jehiel, "Equilibrium on a Traffic Corridor with Several Congested Modes," Transportation Science 27, 16-24 (1993).

12. K.L. Judd, "The Law of Large Numbers with a Continuum of IID Random Variables," Journal of Economic Theory 35, 19-25 (1985).

13. H. Konishi, M. Le Breton, and S. Weber "Equilibrium in a Model with Partial Rivalry," Journal of Economic Theory 72, 225-237 (1997a).

14. H. Konishi, M. Le Breton, and S. Weber "Pure Strategy Nash Equilibrium in a Group Formation Game with Positive Externalities," Games and Economic Behavior 21, 161-182 (1997b).

15. M. Kraus and Y. Yoshida, "The Commuter's Time-of-Use Decision and Optimal Pricing and Service in Urban Mass Transit," Journal of Urban Economics 51, 170-195 (2002).

16. A. Mas-Colell, "On a Theorem of Schmeidler," Journal of Mathematical Economics 13, 201-206 (1984).

17. I. Milchtaich, "Congestion Games with Player-Specific Payoff Functions," Games and Economic Behavior 13, 111-124 (1996).

18. I. Milchtaich, "Generic Uniqueness of Equilibrium in Large Crowding Games," Mathematics of Operation Research 25, 349-364 (2000). 
19. I. Milchtaich, "Network Topology and the Efficiency of Equilibrium," Bar-Ilan University, Working Paper 12-01 (2001).

20. A. Nagurney, Network Economics, (Kluwer), Boston (1993).

21. G.F. Newell, "The Morning Commute for Non-identical Travelers," Transportation Science 21, 74-88 (1987).

22. T. Quint and M. Shubik, "A Model of Migration," Yale University, Cowles Foundation Discussion Paper (1994).

23. D.K. Richter, J. Griffin, and R. Arnott, "Dynamic User Equilibria on a Simple Congested Transportation Network with Heterogenous Commuters," Boston College, Working Paper (1992).

24. R.W. Rosenthal, "A Class of Games Possessing Pure-Strategy Nash Equilibrium," International Journal of Game Theory 2, 65-67 (1973).

25. W.H. Sandholm, "Potential Games with Continuous Player Sets," Journal of Economic Theory 97, 81-108 (2001a).

26. W.H. Sandholm, "Evolutionary Implementation and Congestion Pricing," forthcoming in Review of Economic Studies (2001).

27. D. Schmeidler, "Equilibrium Points of Nonatomic Games," Journal of Statistical Physics 7, 295-300 (1973).

28. Y. Sheffi, Urban Transportation Networks - Equilibrium Analysis with Mathematical Programming Methods, Prentice-Hall, Englewood Cliffs, New Jersey (1985). 
29. K. Small and J. Yan, "The Value of 'Value Pricing' of Roads: Second-Best Pricing and Product Differentiation," Journal of Urban Economics 49, 310-336.

30. M.J. Smith, "The Existence of a Time-Dependent Equilibrium Distribution of Arrivals at a Single Bottleneck," Transportation Science 18, 385-394.

31. T. Tabuchi and D.-Z. Zeng, "Stability of Spatial Equilibrium," University of Tokyo, Working Paper (2001).

32. E.T. Verhoef and K.A. Small, "Product Differentiation on Roads: Second-Best Congestion Pricing with Heterogeneity under Public and Private Ownership," University of California at Irvine, Working Paper (1999).

33. W.S. Vickery, "Congestion Theory and Transport Investment," American Economic Review (Papers and Proceedings) 59, 251-260 (1969).

34. B.W. Wie, "A Differential Game Approach to the Dynamic Mixed Behavior Traffic Network Problem," European Journal of Operations Research 83, 117136 (1995). 